\title{
Targeting histone deacetylase 8 as a therapeutic approach to cancer and neurodegenerative diseases
}

\begin{abstract}
Histone deacetylase 8 (HDAC8), a unique class I zinc-dependent HDAC, is an emerging target in cancer and other diseases. Its substrate repertoire extends beyond histones to many nonhistone proteins. Besides being a deacetylase, HDAC8 also mediates signaling via scaffolding functions. Aberrant expression or deregulated interactions with transcription factors are critical in HDAC8-dependent cancers. Many potent HDAC8-selective inhibitors with cellular activity and anticancer effects have been reported. We present HDAC8 as a druggable target and discuss inhibitors of different chemical scaffolds with cellular effects. Furthermore, we review HDAC8 activators that revert activity of mutant enzymes. Isotype-selective HDAC8 targeting in patients with HDAC8-relevant cancers is challenging, however, is promising to avoid adverse side effects as observed with pan-HDAC inhibitors.
\end{abstract}

First draft submitted: 1 June 2016; Accepted for publication: 19 July 2016;

Published online: 30 August 2016

Keywords: cancer $\bullet$ druggable $\bullet \mathrm{HDAC} \bullet$ hydroxamic acid $\bullet$ inhibitor $\bullet \mathrm{SMC}$ • stem cell - T-cell • therapy

The past decade has witnessed an increased attention to the development of targeted cancer therapies. The vast understanding of the molecular dynamics in cancer cells and their difference in normal cells has triggered thorough investigations of 'cancer-specific molecular targets' and treatment strategies [1]. Besides compounds that target specific oncogenes, small molecules, which influence gene transcription, are currently in the limelight as new modalities for cancer therapy. Examples for drugs of this category are histone deacetylase (HDAC) inhibitors, such as vorinostat suberoylanilide hydroxamic acid (SAHA), the first HDAC inhibitor to be approved for clinical use by US FDA for the treatment of refractory cutaneous T-cell lymphoma [2].

HDACs remove acetate from acetylated $\varepsilon$-amino groups of lysines in histones. Removal of acetyl groups causes global condensation of chromatin and suppression of gene expression [3] and HDACs play an important role in epigenetic regulation. Such epigenetic modifiers, called 'erasers' [3], often occur in multiprotein complexes [4]. HDACs are also termed lysine deacetylases (KDACs), since these enzymes also remove acetyl groups from lysines of numerous nuclear and cytosolic proteins, affecting both gene transcription and cellular signaling $[5,6]$. Disturbances in HDAC normal expression or activity result in aberrant cellular functions [7], thus, associating them with a multitude of diseases [8]. Therefore, HDACs are emerging as stimulating molecular targets for therapeutic intervention, including cancer.

Four classes of HDACs are known, classes I-IV that constitute 18 different isotypes which are either zinc (classical HDACs: I, II and IV) or nicotinamide adenine dinucleotide (NAD, Sirtuins: III) dependent [9]. All the FDA-approved HDAC inhibitors are broad-spectrum HDAC inhibitors, hitting more than one of the isotypes. Because of
Alokta Chakrabarti', Jelena Melesina², Fiona R Kolbinger ${ }^{3}$, Ina Oehme ${ }^{3}$, Johanna Senger ${ }^{1}$, Olaf Witt ${ }^{3,4}$, Wolfgang Sippl ${ }^{2}$

\& Manfred Jung $\star^{* 1,5}$

${ }^{1}$ Institute of Pharmaceutical Sciences, Albert-Ludwigs-Universität Freiburg, Freiburg, Germany

2Institut für Pharmazie, Martin-LutherUniversität Halle-Wittenberg, Halle, Germany

${ }^{3}$ Clinical Cooperation Unit Pediatric Oncology, German Cancer Research Centre (DKFZ), Heidelberg, Germany ${ }^{4}$ Department of Pediatric Oncology, Hematology \& Immunology, University Hospital Heidelberg, Heidelberg, Germany ${ }^{5}$ German Cancer Consortium (DKTK),

Freiburg, Germany

*Author for correspondence:

Tel: +497612034896

Fax: +497612036321

manfred.jung@pharmazie.uni-freiburg.de 
the associated adverse side effects of pan inhibitors, recently, the focus has shifted to specific isotypes. HDAC8, a class I isotype, is gaining importance due to its prominent role in specific cancer subtypes as T-cell lymphoma, childhood neuroblastoma and other ailments as X-linked intellectual disability and parasitic infections [10-13].

HDAC8 is a $\mathrm{Zn}^{2+}$-dependent class I HDAC, identified almost 15 years ago as a $42 \mathrm{kDa}$ protein containing 377 amino acids (aa) [14-16]. Found both in nucleus and cytoplasm [17,18], many of the cellular deacetylation targets of HDAC8 identified so far are known to localize into the nucleus (e.g., AT-rich interactive domain-containing protein $1 \mathrm{~A}$, estrogen receptor alpha, hEST1B and structural maintenance of chromosome 3 [SMC3]) [19-22]. It is an X-linked protein in human [14] and had diverged early from other class I members implying significant functional specialization [9]. Indeed, the independence of cofactors for activity and the absence of the protein binding domain reflect specific characteristics of HDAC8 not present in other members of this class [14-16]. Some active site features such as the highly flexible L1 loop, the conserved aspartate 101 and a regulation by serine 39 phosphorylation add to the discrete functional specialization of HDAC8 [23,24]. Hence, mutations to HDAC8 active site lead to loss of activity and are for example, linked with Cornelia de Lange syndrome (CdLS) $[19,25,26]$.

One fundamental epigenetic role of HDAC8 is the control of the skull morphogenesis. Haberland et al. showed that deletion of HDAC8 leads to perinatal lethality in mice due to skull instability [27]. Further fascinating roles of HDAC8 are still in discovery. Whether in bone differentiation [28] or repression of interleukin $\beta$ [29] or toxin-induced resistance of macrophages [30], HDAC8 reveals novel functions in each case. HDAC8 has been implicated in viral infections [31] and in parasitic disease models HDAC8 inhibition reduced infection load $[13,32]$. Very distinctively, HDAC8 is correlated to T-cell lymphoma, childhood neuroblastoma and gastric cancer $[33,34]$. This review aims to discuss the therapeutic intervention of HDAC8 in cancer and the available inhibitors for isotype specific targeting in HDAC8 relevant ailments. A short section on HDAC8 activators is also presented.

\section{Deacetylase activity \& interaction partners}

Although histones are viewed as the classical HDAC substrates, there is ample proof on nonhistone substrates $[3,6]$. For HDAC8, there is conflicting evidence concerning histones as bonafide in vivo deacetylation substrates [35]. While there is data that hyperacetylation results as a consequence of HDAC8 inhibitor treatment [36] or overexpression leads to low acetyla- tion [15,37], sophisticated MS analyses have failed to detect histones as HDAC8 substrates [22,38]. There is a possibility that HDAC8 might prefer deacetylation of only very particular sites, which could be masked by global acetylation patterns [39] or likely be dependent on the cell type [40]. However, in vitro, deacetylation of histone variants like $\mathrm{H} 2 \mathrm{~A} / \mathrm{H} 2 \mathrm{~B}, \mathrm{H} 3$ and H4 by HDAC 8 has been observed [14,16]. Even short peptides derived from histones are excellent in vitro substrates and knowledge on deacetylation sites in such peptides has contributed to the understanding of sequence specificity for HDAC8 deacetylation sites, for example, deacetylation occurs at lysines 14, 16, 20 on histone H4-derived peptides [14,16,41] and $\mathrm{K}(\mathrm{Ac}) \mathrm{RHR}$ is a preferred motif in this histone subtype [42].

Besides histones, a large number of nonhistone candidates have been recognized as substrates or interaction partners of HDAC8. SMC3 [19], ERR $\alpha$ [20] and p53 [43] have been shown by RNAi or pharmacological inhibition of HDAC8 to be direct cellular substrates with clear concentration-dependent hyperacetylation effects. Mass spectrometric analysis of cellular lysates after treatment with HDAC8 inhibitors has established several novel proteins as retinoic acid-induced 1 , zinc-finger Ran binding domain-containing protein 2, AT-rich interactive domain-containing protein $1 \mathrm{~A}$, nuclear receptor coactivator 3 and thyroid hormone receptor-associated protein 3 as HDAC8 deacetylation targets [22]. Very recently, four new members have joined the known substrates: peroxiredoxin 6 , phoshoglycerate mutase 1 , high mobility group protein B1 and Parkinson protein 7 [38]. Peptide arrays have revealed an even broader range of acceptable range of substrates, indicating further physiological substrates to be discovered [44]. Other proteins, such as inv(16) fusion protein [45], CREB [46], DEC1 [47], Hsp20 [48], human ever-shorter telomeres 1B (hEST1B) [21] and $\alpha$-actin [18] have been found to be associated with HDAC8. However, it is unclear whether these proteins are direct acetylation targets or form a part of complex in which HDAC8 acts as a scaffold. For instance, HDAC8 coimmunoprecipitated with both CREB and protein phosphatase 1; ectopic expression of HDAC8 decreased CREB activity [46]. Similarly, HDAC8 colocalized and immunoprecipitated with smooth muscle myosin heavy chain and possibly connects to inv(16) fusion protein via this domain [45]. In this case, pharmacological inhibition with trichostatin A affected transcriptional activities of the $\operatorname{inv}(16)$ fusion protein [45]. Both for CREB and inv(16), such evidence suggests HDAC8 can interact with these proteins as deacetylation substrate or as members of a cooperative complex. More clues for scaffolding function come from interactions with $\alpha$-actin [18]. Colocalization of 
HDAC8 with $\alpha$-actin and subsequent knockdown effects in human smooth muscle cells (including phenotypic changes such as smaller cells with loss of contractibility or spreading) without changes in global $\alpha$-actin acetylation points toward a scaffolding behavior of HDAC8 rather than deacetylase activity in those cases [49]. Furthermore, DNA-ChIP analysis demonstrated a colocalization of HDAC8 with the transcription factor DEC1 [47]. Modulation of HDAC8 expression affected DEC1- and DEC1-regulated TAp73 transcription factor [47] implying a recruiting role of DEC-1 with HDAC8, but, it remains unclear whether HDCA8 catalyzes the deacetylation of DEC1. In a cellular context, dissection of deacetylation and scaffolding functions for HDAC8 is difficult. Most probably, both functionalities are intertwined and studies with catalytically dead mutants could provide mechanistic clues about the mystery behind HDAC8 physiology. However, the likelihood that many HDAC8 substrates could be shared by other HDACs complicates such mechanistic approaches. Moreover, nonepigenetic roles of HDAC8 are also possible. Recent studies demonstrating an ability of HDAC8 to control Notch stability [50] or binding to miRNA miR-216b [34] point to an ever-increasing complexity of the role of HDAC8 in cells.

\section{HDAC8 structure \& inhibitors}

Association of HDAC8 with a large number of diverse proteins (stated previously) leaves no doubt that it mediates crucial signaling events in human pathophysiology. Considering the wide range of interaction partners, HDAC8 is a key enzyme in cancer, CdLS, virus and parasite infection (see review [51]), pointing out its great potential as a therapeutic target. This raises the question whether we can create drug molecules to selectively inhibit the enzyme and envisage a cure for the subset of diseases that depend on HDAC8 activity. For this purpose, a closer look at HDAC8 crystal structure sheds light on the druggability of this HDAC isotype.

Currently (end of 2015) 50 x-ray structures of human HDACs and six structures of Schistosoma mansoni HDAC8 are stored in the Protein Data Bank, giving structural insight into zinc-dependent HDACs (Table 1). This information allows the comparison between HDAC8 and other HDAC isotypes. Structurally, HDAC8 has conserved features similar to those of other representatives of the family. First of all, it shares a common architecture of the HDAC catalytic domain, the core of which consists of eight-stranded $\beta$-sheets surrounded by $\alpha$-helices. These secondary structure elements are connected by loops [35,52], seven of which form the substrate binding pocket with the zinc ion at the bottom [53], where all cocrystallized inhibitors bind [52] (Figure 1A). Second, the binding pocket of HDAC8 has conserved residues [52], which are observed among all currently solved crystal structures of HDACs and adopt highly similar side chain conformations. In particular, the zinc ion coordinating residues (corresponding to D178, D267 and H180 in hHDAC8), two histidines, coordinating the hydroxamic acid of SAHA in hHDAC8 PDB ID 1T69 (H142 and H143) and phenylalanine forming the wall of the lysine binding channel (corresponding to F208 in hHDAC8) are all conserved [53] (Figure 1B). The structural similarities of HDACs explain their preferences to similar ligands and pose a major challenge to the design of HDAC8-selective compounds.

Fortunately, HDAC8 exhibits a number of unique characteristics allowing the development of selective inhibitors. This makes HDAC8 an attractive target for structure-based drug design. One particularly interesting feature in this regard is the formation of the foot pocket (also called acetate release channel). This subpocket is observed in class I HDACs (HDAC1, 2, 3 and 8), but not in class II isotypes (HDAC4 and 7) $\mathrm{x}$-ray structures. Its presence or absence is caused by a different conformation of the loop L3 and HDACclass specific differences in the aa composition in these two classes of HDACs, as shown for HDAC8 and HDAC7 in Figure 1C. This binding pocket shape difference explains why class I HDACs are able to accommodate ligands with large scaffolds occupying the foot pocket (like substituted ortho-anilinobenzamides or aa derivatives), while class IIa HDACs prefer compact groups, filling the small cavity near the zinc ion (trifluoromethylketones, trifluoromethyloxadiazoles) [54]. In all $\mathrm{x}$-ray structures of HDAC4 and HDAC7 the entrance to the foot pocket is closed due to alternative conformation of the loop L3 site, preceding the conserved histidines discussed previously (H142 and H143 in hHDAC8) (Figure 1C). This part of the loop is formed by the same residues (glycine and two prolines) in all class IIa HDACs, suggesting that probably none of them form the foot pocket. Of note, S. mansoni HDAC8 has structural differences, allowing for species-selective HDAC8 inhibitors [13,32]. Comparison of the gatekeeper residues, opening the foot pocket in human class I HDACs, shows that HDAC8 stands out from the other representatives. Instead of the leucine residue (corresponding to L144 in hHDAC2) observed in HDAC1, 2 and 3, HDAC8 has a bulkier tryptophan residue (W141). This aa substitution changes the shape of the pocket (Figure 1D) and causes preferences to different ligands.

Another characteristic structural feature of HDAC8 is the presence of the side pocket. Both human and $S$. 

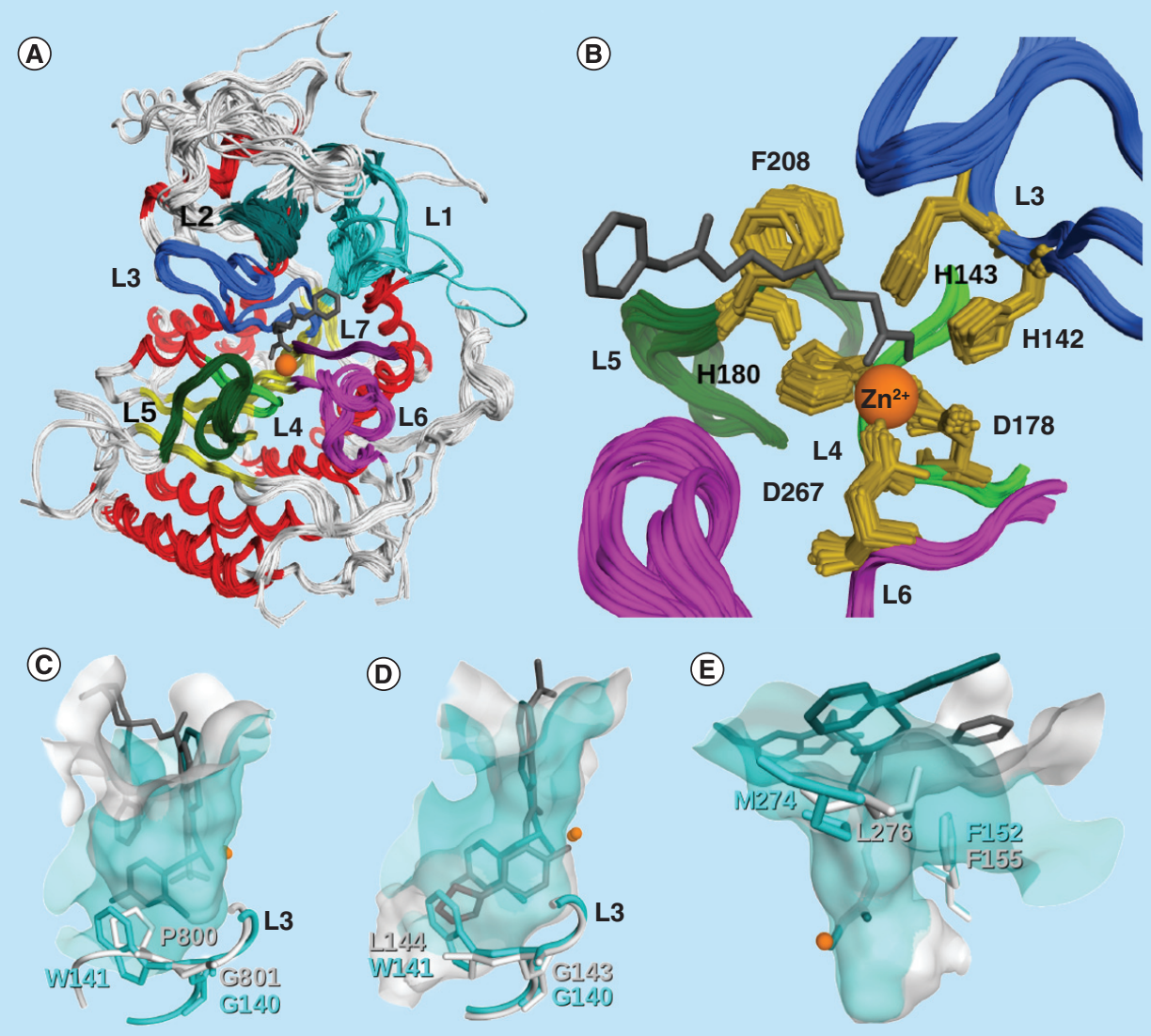

Figure 1. Histone deacetylase 8 crystal structures. Similarities (A, B) and differences (C-E) between HDAC8 and other histone deacetylases. (A \& B) Superimposed all currently available 56 x-ray structures of human and parasitic HDACs (hHDAC1, hHDAC2, hHDAC3, hHDAC8, smHDAC8, hHDAC4 and hHDAC7). The zinc ion (orange ball) and the ligand (SAHA, gray sticks) are shown for hHDAC8 PDB ID 1 T69 to define the binding pocket. (A) Common catalytic domain in all HDACs, built by conserved $\beta$-sheet (yellow ribbons) surrounded by $\alpha$-helices (red ribbons) and flexible loops. Loops (or their parts), forming the binding pocket, are colored (L1 - cyan, L2 - turquoise, L3 - blue, L4 - lime, L5 - dark green, L6 - magenta, L7 - deep purple) and the others are white. (B) Conserved binding pocket residues (dark yellow sticks) are adopting similar side chain conformations in different HDACs. The numbers of residues are given only for hHDAC8 for clarity. The loops, bearing the conserved residues are shown as ribbons and colored in the same way as in (A): L3 - blue, L4 - lime, L5 - dark green and L6 - magenta. (C-E) Differences between HDAC8 (cyan residues, L3 and surface of the binding pocket, turquoise ligand) and other HDAC isotypes (white residues, L3 and surface of the binding pocket, gray ligand). Zinc ions are shown as orange ball. Only residues, changing the binding pocket shape dramatically are shown for clarity. (C) Comparison of hHDAC8 (PDB ID 3SFH) and hHDAC7 (PDB ID 3ZNS) - foot pocket in hHDAC8 is opened by the movement of the gatekeeper residue W141, while in hHDAC4 it is closed because of the different orientation of the L3. (D) Comparison of hHDAC8 (PDB ID 3SFH) and hHDAC2 (PDB ID $4 L Y 1$ ) - foot pocket is opened in both isotypes and the orientation of $L 3$ is the same, but the gatekeeper residue in hHDAC8 (W141) is bigger than in hHDAC2 (L144), which restricts the space in this area. (E) Comparison of hHDAC8 (PDB ID 2V5X) and hHDAC2 (PDB ID 4LXZ) - side pocket is observed in hHDAC8, but is closed in hHDAC2, which is caused by a closer proximity of the gatekeeper residues in hHDAC2 (F155 and L276) than in hHDAC8 (F152 and M274). HDAC: Histone deacetylase; SAHA: Suberoylanilide hydroxamic acid.

mansoni HDAC8 demonstrate opening of this subpocket, while in other class I HDACs (hHDAC1-3) it is not observed [53]. This explains the preference of HDAC1-3 to rod-shaped SAHA-like ligands with extended alkyl chain linkers, while HDAC8 is more likely inhibited by compounds with bulky linkers (e.g., phenylene spacers), which do not fit well to the narrow binding pocket of the HDAC1-3 isotypes $[10,55,56]$. A comparison between $\mathrm{x}$-ray structures of hHDAC8 and hHDAC2 shows the structural reason of the side pocket formation. Due to a different conformation of the loop L6, the gatekeeper residues in hHDAC8 (F152 and M274) are placed more apart than in hHDAC2 (F155 and L276), causing the formation of the cavity (Figure 1E). To conclude, the comparison of available crystal structures of different HDACs indicates that HDAC8 has a number of unique features discriminating it from other isotypes and making it a 
druggable target with the possibility to create potent and isotype-selective inhibitors.

\section{Role of HDAC8 in oncogenesis}

HDAC8 appears to be an attractive anticancer target, since in some tumor entities HDAC8 seems to play an explicit tumor relevant role (Table 2). Specifically, HDAC8 is expressed in colon, breast, lung, hepatocellular carcinoma, gastric cancer, pancreas tumor tissue, metastatic melanoma, acute myeloid leukemia (AML), acute lymphocytic leukemia (ALL) as well as in childhood tumors of the nervous system, such as neuroblastoma (Figure 2) [33,43,57-59]. Although HDAC8 is also expressed in corresponding normal tissue, it is by trend higher expressed in cancer tissues [43,57] and displays a significant upregulation in aggressive stage 4 neuroblastomas [58]. In invasive breast tumor cells, HDAC8 is among the three HDAC family members that are upregulated and driving invasiveness [60]. Vice versa, the RNAi-mediated knockdown of HDAC8 expression in human lung, colon, leukemia, gastric adenocarcinoma and cervical cancer cell lines inhibits tumor cell proliferation [33,61,62]. Upregulation of HDAC8 promotes proliferation and inhibits apoptosis in hepatocellular carcinoma [43] and gastric cancer [33]. HDAC8 expression itself can be regulated by the lipogenic transcription factor sterol regulatory element binding protein-1 and the SOX-family of transcription factors. SREBP-1 links lipid metabolism, insulin resistance and cancer development and has been shown to directly upregulate HDAC8 in models of nonalcoholic fatty liver disease-associated hepatocellular carcinoma [40]. Microarray analyses of adult T-cell leukemia/lymphoma revealed that HDAC8 expression in these tumor cells is regulated by SOX4, which directly activates the HDAC8 promoter [62]. SOX4 is a transcription factor, which is required for B-lymphocyte development [63], but also during development of the sympathetic nervous system $[64,65]$.
So far, several mechanisms of action are described for HDAC8. In colon cancer, for example, the Bcl-2-modifying factor $(B M F)$ has been identified to be a direct target gene of HDAC8 repression and HDAC8 derecruitment is sufficient to activate the target gene [73]. Furthermore, STAT3 associates and cooperates with HDAC8 to repress $B M F$ transcription [73]. BMF has an important function in the execution of apoptosis triggered by the HDAC inhibitory metabolite methylselenopyruvate, which is a competitive inhibitor of HDAC8 [73,74]. In addition, HDAC8 has been implicated in contributing to tumorigenesis by regulating telomerase activity [21]. In AML, the inv(16) fusion protein associates with HDAC8 in order to repress the transcription of AML1-regulated genes [45]. Several studies describe a link between HDAC8 and the tumor suppressor $\mathrm{p} 53$. The interaction of inv(16) with HDAC8, for example, causes HDAC8-mediated deacetylation and inactivation of $\mathrm{p} 53$ in AML leukemia stem cells which promotes tumor cell transformation [69]. Yan et al. discovered that the knockdown of HDAC8 results in repression of HoxA5 and consequently to decreased HoxA5-dependent expression of wild-type as well as mutant p53 [82]. p53 expression can be rescued by enforced expression of HoxA5. Conversely, the ectopic expression of HDAC8 enables enhanced transcription of p53 [82]. As HDAC8 is required for $\mathrm{p} 53$ expression, regardless whether wildtype or mutated, inactivation of HDAC8 may be more effective in tumor cells harboring a p53 mutation. Results with the HDAC8 activator TM-2-51 illustrate another level of complexity. The treatment of $\mathrm{p} 53$ wildtype SH-SY5Y neuroblastoma cells with the activator induces apoptosis in these cells, whereas BE(2)-C neuroblastoma cells, harboring mutated p53 [83] respond well to HDAC8 inhibitor treatment with growth arrest and differentiation [77]. This supports the hypothesis that activation of HDAC8 might be profitable

\section{Table 1. Resolved crystal structures of histone deacetylases from human and Schistosoma mansoni.}

\begin{tabular}{|c|c|c|c|}
\hline Organism & HDAC & PDB ID & Number of x-ray structures \\
\hline \multirow[t]{5}{*}{ Human } & hHDAC1 & $4 B K X$ & 1 \\
\hline & hHDAC2 & $3 M A X, 4 L X Z, 4 L Y 1$ & 3 \\
\hline & hHDAC8 & $\begin{array}{l}\text { 1T64, 1T67, 1T69, 1VKG, 1W22, 2V5W, 2V5X, 3EW8, 3EWF, } \\
\text { 3EZP, 3EZT, 3F06, 3F07, 3F0R, 3MZ3, 3MZ4, 3MZ6, 3MZ7, } \\
\text { 3RQD, 3SFF, 3SFH, 4QA0, 4QA1, 4QA2, 4QA3, 4QA4, 4QA5, } \\
\text { 4QA6, 4QA7, 4RN0, 4RN1, 4RN2 }\end{array}$ & 32 \\
\hline & hHDAC4 & 2VQJ, 2VQM, 2VQO, 2VQQ, 2VQV, 2VQW, 4CBT, 4CBY & 8 \\
\hline & hHDAC7 & $3 \mathrm{COY}, 3 \mathrm{COZ}, 3 \mathrm{C} 10,3 \mathrm{ZNR}, 3 \mathrm{ZNS}$ & 5 \\
\hline Schistosoma mansoni & smHDAC8 & 4BZ5, 4BZ6, 4BZ7, 4BZ8, 4BZ9, 4CQF & 6 \\
\hline
\end{tabular}


(A)

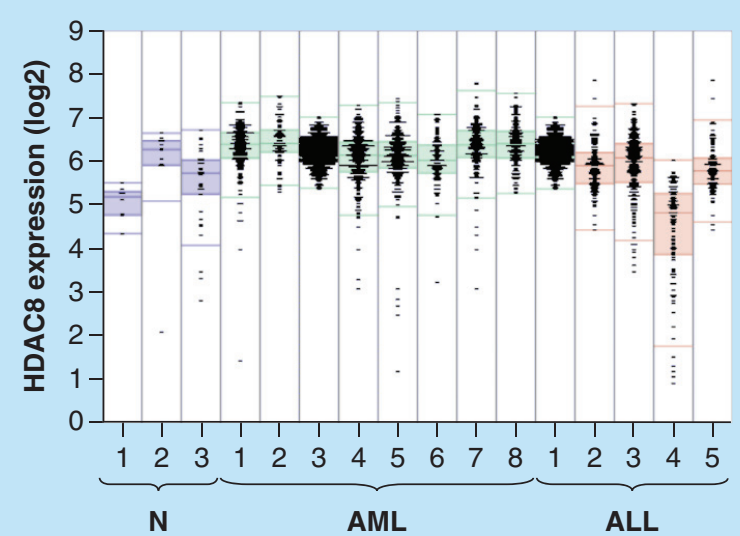

(C)

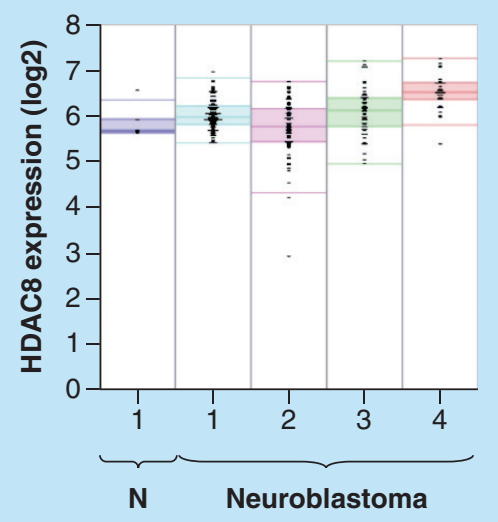

(B)

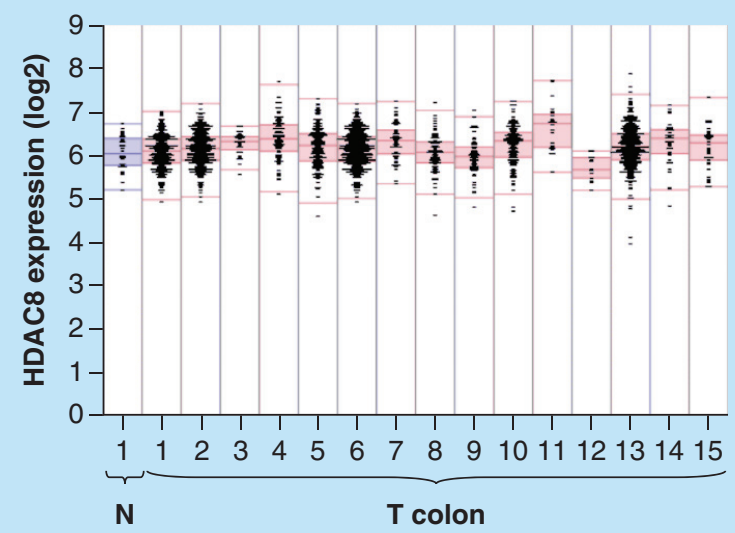

(D)

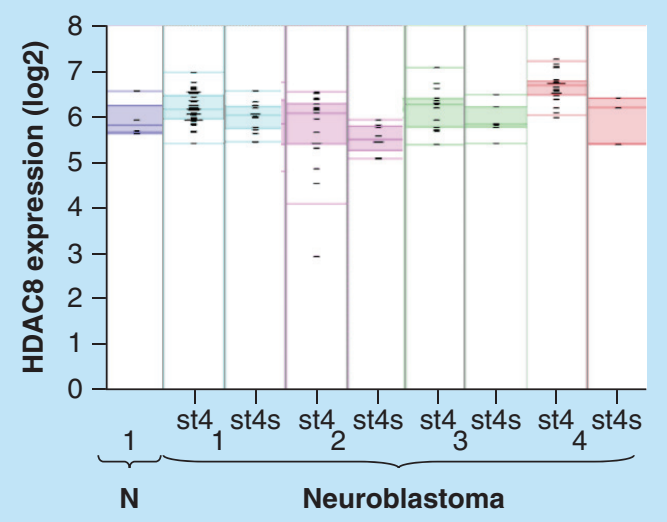

Figure 2. Histone deacetylase 8 expression in normal and tumor cells. R2 MegaSampler [66] with HDAC8 probe 223345_at. (A) HDAC8 expression of normal B cells (N1-2, blue), leukocytes (N3, blue) and expression in AML (1-8, green) and ALL (1-5, orange). (B) Normal (blue) colon (N1) expression compared with expression in colon cancer ( $T$ Colon 1-15, red). (C) Normal neural crest (N1, blue) expression compared with expression in neuroblastoma (1-4). (D) Normal neural crest (N1, blue) expression compared with expression in neuroblastoma INSS stage 4 and stage 4s samples of the Versteeg (1), Delattre (2), Hiyama (3) and Latowska (4) cohort.

ALL: Acute lymphocytic leukemia; AML: Acute myeloid leukemia; HDAC8: Histone deacetylase 8.

under p53 wild-type conditions, whereas inhibition of HDAC8 is more reasonable in tumors carrying a mutant $\mathrm{p} 53$ gene. However, further studies are necessary to fully unravel the mechanistic link, since some tumor entities do not follow that model. For example, knockdown of HDAC8 expression elevates the expression and acetylation of $\mathrm{p} 53$ in hepatocellular carcinoma cells, resulting in decreased cell proliferation and activation of apoptosis $[40,43]$. Additionally, further mechanistic studies are necessary to fully understand the link of the p53-HDAC8-HoxA5 axis, initially described for adult cancer cells [82], in childhood neuroblastoma. Besides the regulation of $\mathrm{p} 53$, HDAC8 is also involved in the regulation of another transcription factor of the p53 family, p73. This transcription factor also plays a key role in many biological processes, such as neuronal development and tumorigenesis [84]. The trans- activating isoform TAp73 has prodifferentiating roles and induces cell cycle arrest as well as apoptosis. In contrast, the aminoterminal truncated $\Delta \mathrm{Np} 73$ isoform has prosurvival functions and favors oncogenic transformation [85]. HDAC8 is required for the transcription factor DEC1 to enhance TAp73 expression [47]. DEC1 interacts with HDAC8 and recruits HDAC8 to the TAp73, but not the $\triangle \mathrm{Np} 73$, promoter [47]. DEC1 in turn, is a target of the p53 family [86].

\section{HDAC8 inhibitors as anticancer therapeutics}

The specific involvement of HDAC8 in cancers such as leukemia and childhood neuroblastoma indicates great therapeutic potential. Especially in neuroblastoma and T-cell lymphoma, both entities with a clear correlation of HDAC 8 activity with the disease, specific targeting of HDAC8 might present a rationale to 


\section{Table 2. Histone deacetylase 8 expression in cancer.}

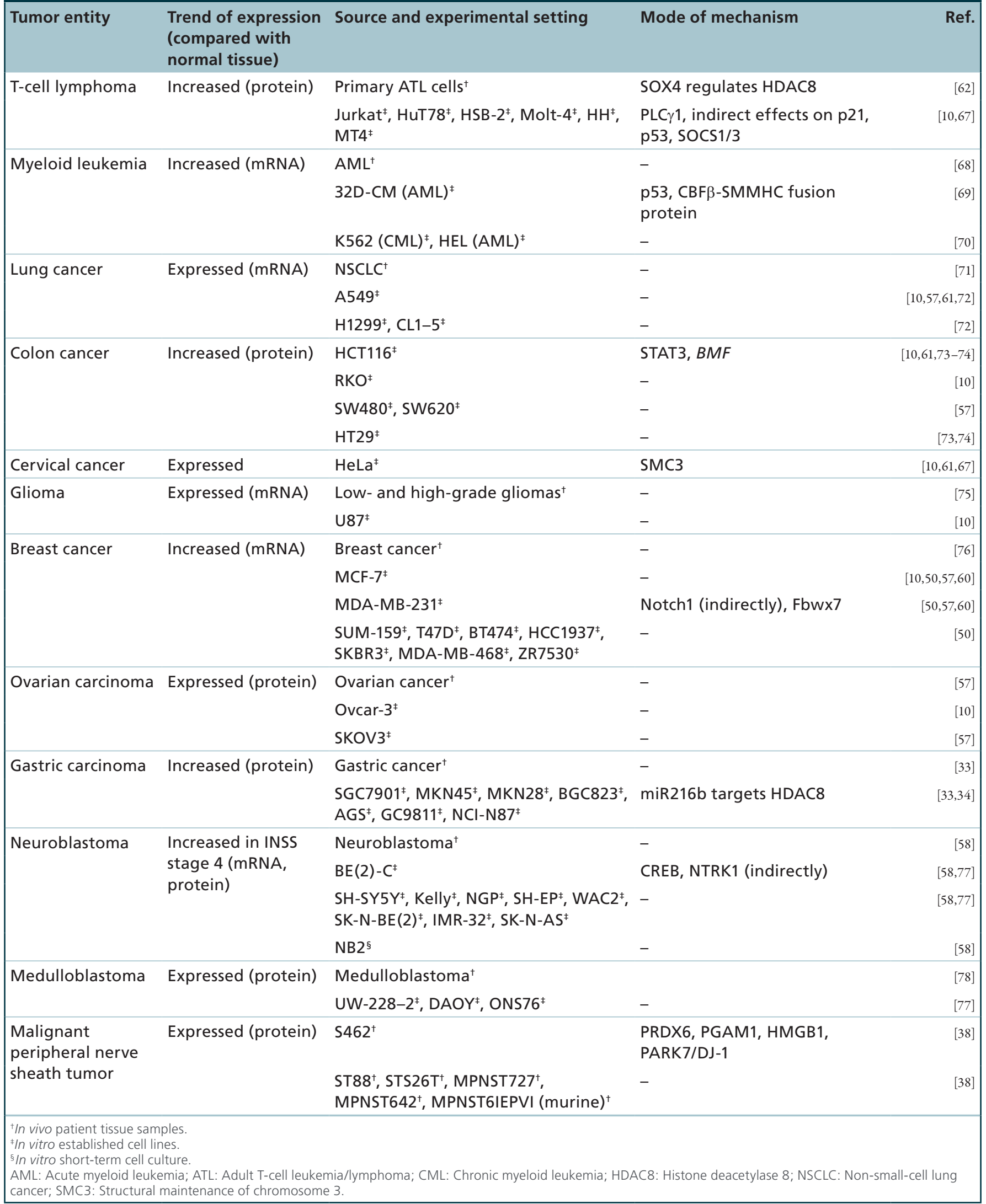


Table 2. Histone deacetylase 8 expression in cancer (cont.).

\begin{tabular}{|c|c|c|c|c|}
\hline Tumor entity & $\begin{array}{l}\text { Trend of expression } \\
\text { (compared with } \\
\text { normal tissue) }\end{array}$ & Source and experimental setting & Mode of mechanism & Ref. \\
\hline \multirow{4}{*}{$\begin{array}{l}\text { Hepatocellular } \\
\text { carcinoma }\end{array}$} & \multirow[t]{4}{*}{ Increased (protein) } & \multicolumn{2}{|l|}{ Hepatocellular carcinoma§ } & {$[40,43]$} \\
\hline & & $\mathrm{HepG}^{\dagger}$ & p53, $\beta$-catenin & {$[40,43]$} \\
\hline & & Bel-7404 ${ }^{+}$ & p53 (Lys382), $\beta$-catenin & {$[40,43]$} \\
\hline & & $\mathrm{PLC}^{+}$ & p53, $\beta$-catenin & {$[40]$} \\
\hline \multirow[t]{2}{*}{ Prostate cancer } & \multirow[t]{2}{*}{ Decreased (protein) } & $\begin{array}{l}\text { Malignant and nonmalignant } \\
\text { prostate tissues }^{\S}\end{array}$ & & {$[57,79]$} \\
\hline & & LNCap $^{\dagger}$, PC-3 ${ }^{\dagger}, \mathrm{DU}_{145^{\dagger}}$ & - & [57] \\
\hline Pancreatic cancer & Expressed (protein) & $\mathrm{MiaPaCa}-2^{\dagger}, \mathrm{PANC}^{-1^{\dagger}}$ & - & [57] \\
\hline Urothelial cancer & Increased (mRNA) & $\begin{array}{l}\text { VM-CUB1 } 1^{\dagger}, \text { RT- } 112^{\dagger}, \text { SW- } 1710^{\dagger}, 639-V^{\dagger}, \\
\text { UM-UC-3 }\end{array}$ & p21 (indirectly) & [80] \\
\hline Uterine cancer & $\begin{array}{l}\text { Expressed (protein), } \\
\text { specific marker of } \\
\text { smooth muscle cell } \\
\text { differentiation }\end{array}$ & 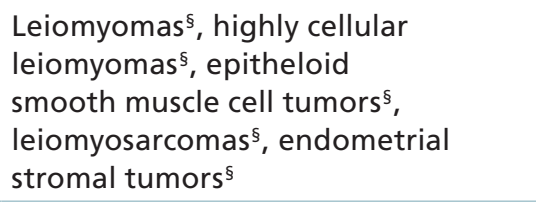 & - & {$[70,81]$} \\
\hline \multicolumn{5}{|c|}{$\begin{array}{l}\text { †In vivo patient tissue samples. } \\
\text { ₹In vitro established cell lines. } \\
\text { \$In vitro short-term cell culture. } \\
\text { AML: Acute myeloid leukemia; ATL: Adult T-cell leukemia/lymphoma; CML: Chronic myeloid leukemia; HDAC8: Histone deacetylase 8; NSCLC: Non-small-cell lung } \\
\text { cancer; SMC3: Structural maintenance of chromosome 3. }\end{array}$} \\
\hline
\end{tabular}

efficacious treatment with limited off-target effects. The FDA-approved broad-spectrum HDAC inhibitor vorinostat (SAHA) which is used to treat refractory cutaneous T-cell lymphoma, is a weaker inhibitor of HDAC8 (micromolar range) than of HDACs 1-3 (nanomolar range). Similarly, trichostatin A completely inhibits HDAC8 activity only at $5 \mu \mathrm{M}$, though in Molt-4 acute lymphoblastic leukemia cells it attenuates HDAC8 expression [87]. Besides the known clinical side effects of SAHA (e.g. leukopenia, thrombopenia, fatigue, diarrhea), this inhibitor has very recently been reported to promote epithelial mesenchymal transition and cell motility in triple negative breast cancer cell culture. This negative side effect was reported to be dependent on HDAC8 [88]. In terms of unspecific side effects, the targeting of one single enzyme seems to be superior to broad spectrum HDAC inhibition when applied in an appropriate tumor entity that displays oncogenic dependency on that particular HDAC family member. In neuroblastoma for example, HDAC8 expression cor- relates with the aggressive tumor stage 4 and thus, with poor outcome. Selective HDAC8 inhibition in neuroblastoma cell lines induces signs of differentiation, such as the outgrowth of neurofilament-positive neurite-like structures [58]. Of note, the targeting of other HDAC family members in this tumor entity affects completely different processes (e.g., apoptosis or autophagy) [89-93]. Consistent with the observation that neural crest-cell-derived neuroblastoma displays oncogenic HDAC8 dependency, HDAC8 controls patterning of the skull in cranial neural crest cells of mice. Consequently, deletion of the gene for HDAC8 in mice leads to perinatal (P1) lethality due to skull instability [27]. Hence, HDAC8 is proposed as a potential new drug target for differentiation therapy by the usage of selective HDAC8 inhibitors to avoid unspecific side effects [11,94].

Hand in hand with the findings of a tumor relevant expression and mode of action of HDAC8, the development and usage of selective HDAC8 inhibitors proceeds. Though, specific inhibitor design is challenging due to 
the flexible structure of this epigenetic modulator, the unique binding pocket of HDAC8 allows the design of HDAC8-selective inhibitors (Figure 3 \& Table 3). So far several specific inhibitors have been reported. Lead structures were identified by either structure-based design, for example, linkerless hydroxamic acids $\left(\mathrm{IC}_{50}\right.$ $0.3 \mu \mathrm{M},>100$-fold selectivity over HDAC1 and 6) [55] or from high-throughput screens like the cyclic thiourea SB379278A $\left(\mathrm{IC}_{50} 0.5 \mu \mathrm{M}\right)$ [95] (Figure 3). However, it remains open which isotype-specific chemical scaffolds would gain preference in follow-up advanced preclini$\mathrm{cal} /$ clinical studies and whether such inhibitors would really be superior to pan-HDAC inhibitors in clinical trials, either for efficacy or toxicity. Within the scope of this review, we discuss the rationale for design of HDAC8-specific inhibitors and present a synopsis of some isotype specific inhibitors of HDAC8 with diverse chemical backbones targeting different mechanistic pathways. For cancer related processes relevant HDAC8 modulators are represented and summarized additionally in Figure 4.

\section{Design of HDAC8-selective inhibitors}

The structural determinants of HDAC8 specificity as discussed above can be addressed by selective inhibitors. For instance, the HDAC8 foot pocket was successfully targeted with two selective small-molecule aa derivatives (PDB ID 3SFF and 3SFH) (Figure 3) [99]. This is a rare example of HDAC inhibitors without a strong chelating function such as hydroxamate, thiol or benzamide. Furthermore, as shown by molecular docking studies, dual HDAC8- and HDAC6-selective meta-substituted benzhydroxamic acids (Figure 3) also take advantage of the specific binding pocket shape of these HDAC isotypes by occupying their side pocket [56].

Due to limited structural information, rational drug design is in many cases is supported by molecular modeling studies. Such computational methods as QSAR modeling [100] and virtual screening [101] have been exploited to search for novel HDAC8 inhibitors. In order to explain structure-activity relationships and to guide the optimization of hit compounds, molecular docking studies are readily used $[32,56,72,96]$. Molecular dynamics simulations help to understand dynamic and mechanistic aspects of HDAC8 behavior [102-105]. Finally, homology modeling of parasitic HDAC8 isotypes sheds light on druggability of those targets and requirements for their inhibitors [53]. It should be noted, however, that one of the main challenges for molecular modeling is the high flexibility of the enzymes, which makes the prediction of the HDACs structure and the binding mode of their inhibitors tricky. A recent analysis of the flexibility of the $S$. mansoni HDAC8 binding pocket based on its crystal structures demonstrates that the shape of the pocket undergoes dramatic changes due to induced fit effects caused by binding of various ligands [53].

Another useful tool in drug design is the structure-activity relationship analysis of already-known inhibitors. This approach can be adopted to understand pharmacophoric features of molecules, which are necessary to gain HDAC8 selectivity. The structural diversity of known small-molecule HDAC8selective inhibitors can be seen in Figure 3. Since all cocrystallized HDAC inhibitors bind to the zinc ion, it is obvious that this interaction is very important to specifically inhibit those enzymes in most cases. Therefore, it is comfortable to classify the HDAC8 inhibitors by the zinc-binding group: hydroxamic acids [10,55,56,67,72,96,98,106-109], aa derivatives [99] and other compounds [95,110]. The hydroxamic acid is a very common and universal metal chelating group, which can effectively bind to any HDAC isotype and to some other metalloenzymes (carbonic anhydrase, matrix metalloproteinase, urease, lipoxygenase, etc.) [11]. Nevertheless, by adjusting the linker of the inhibitor and further substituents it is possible to achieve not only protein family [112], but also isotype selectivity among hydroxamates. From the structures of known HDAC8selective hydroxamate-based inhibitors it can be concluded that meta-substituted benzhydroxamic acids and hydroxamic acids with ortho-substituted cinnamic acid linker are HDAC8 selective. Sometimes they are also equally active on HDAC6 isotype, but in general this $\Gamma$-shape allows gaining selectivity against all other HDAC isotypes [53]. In case of parasubstituted benzhydroxamic acids [113,114] and hydroxamic acids with paraand meta-substituted cinnamic acid linker (panobinostat, belinostat) [115], they usually do not show HDAC8 selectivity with rare exceptions $[55,107]$. The selectivity of SB379278A [95], an HDAC8-inhibitory azetidinone [110] and $\beta$-methylselenopyruvate [74] probably depends on their unusual zinc-binding groups.

\section{HDAC8 inhibitors \\ PCI34051}

PCI34051 (Figure 3 \& Table 3) is probably the most widely used HDAC8-specific inhibitor in research. Based on an indole linker, this hydroxamic acid inhibited HDAC8 with an $\mathrm{IC}_{50}$ of $10 \mathrm{nM}$ and was $>200$ fold selective over HDACs $1 / 6$ and $>1000$-fold over HDACs 2/3/10 [10]. While in seven different solid tumor cell lines PCI34051 did not show any cytotoxic activity (except Ovcar-3 with $\mathrm{GI}_{50} 6 \mu \mathrm{M}$ ), in T-cell-derived tumor cell lines Jurkat (derived from a T-cell leukemia), HuT78 (derived from peripheral T-cell lymphoma), HSB-2 and Molt-4 (both derived from T-ALL) PCI34051 induced cell death with $\mathrm{GI}_{50}$ 
Review Chakrabarti, Melesina, Kolbinger et al.

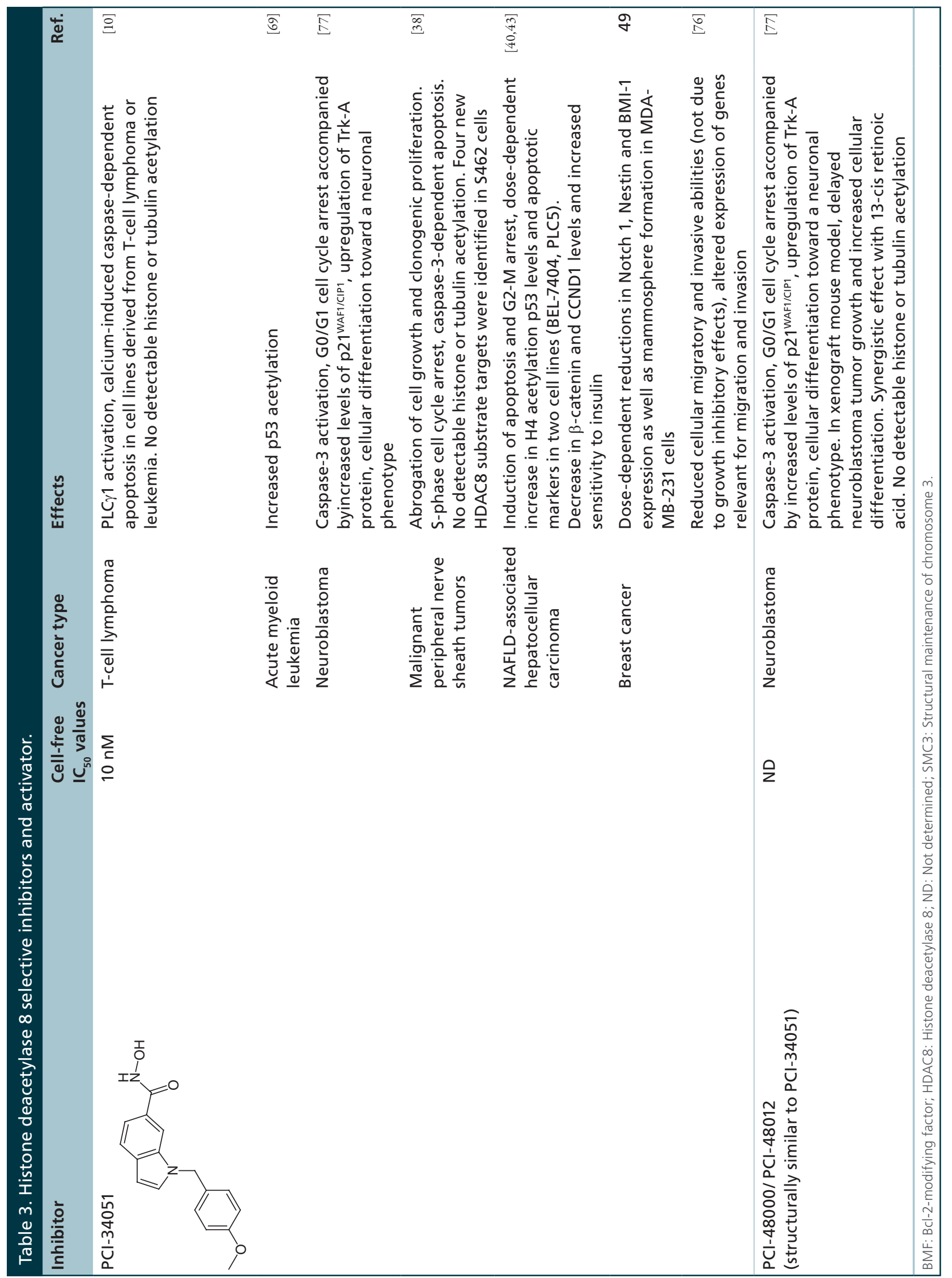




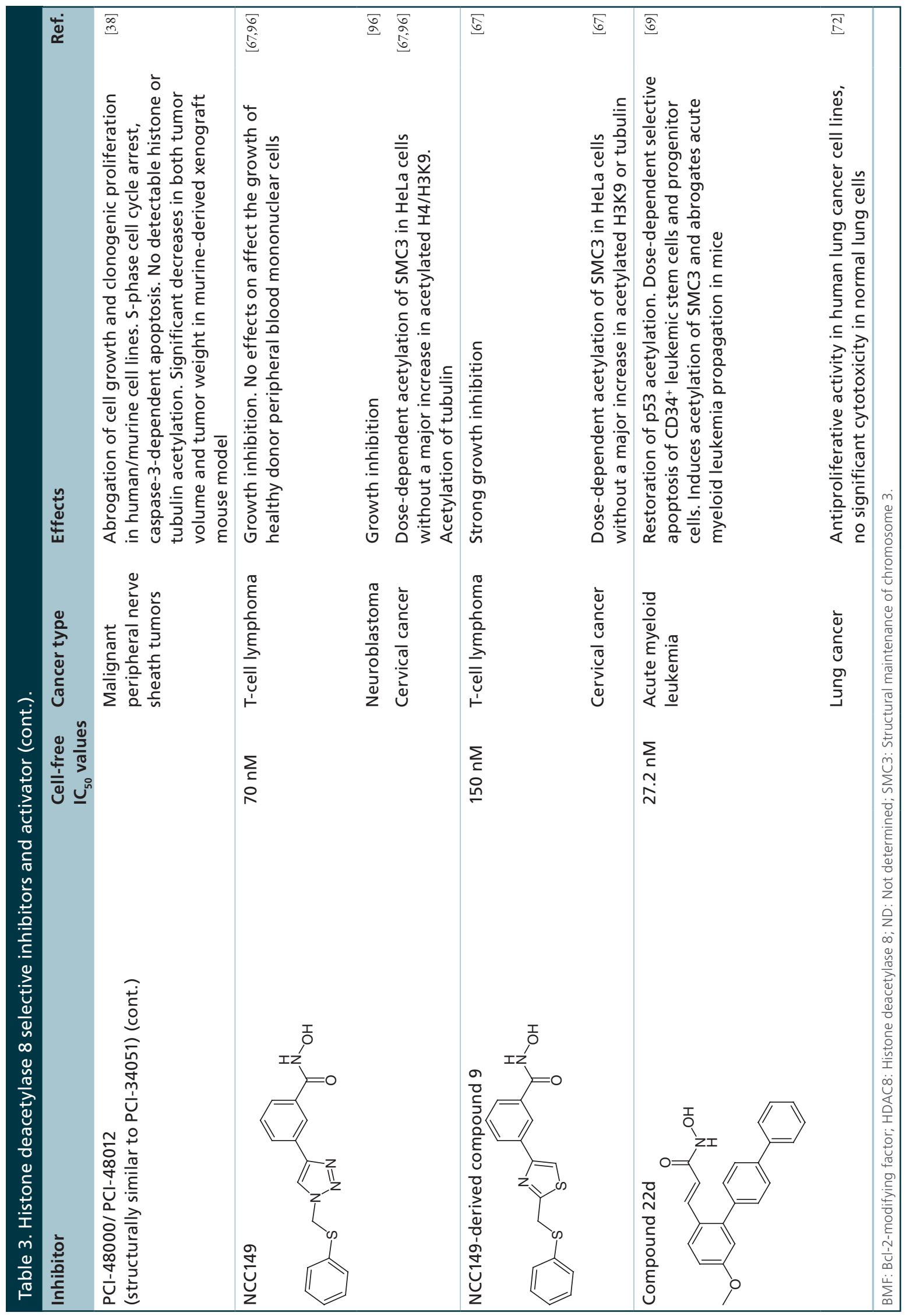


Review Chakrabarti, Melesina, Kolbinger et al.

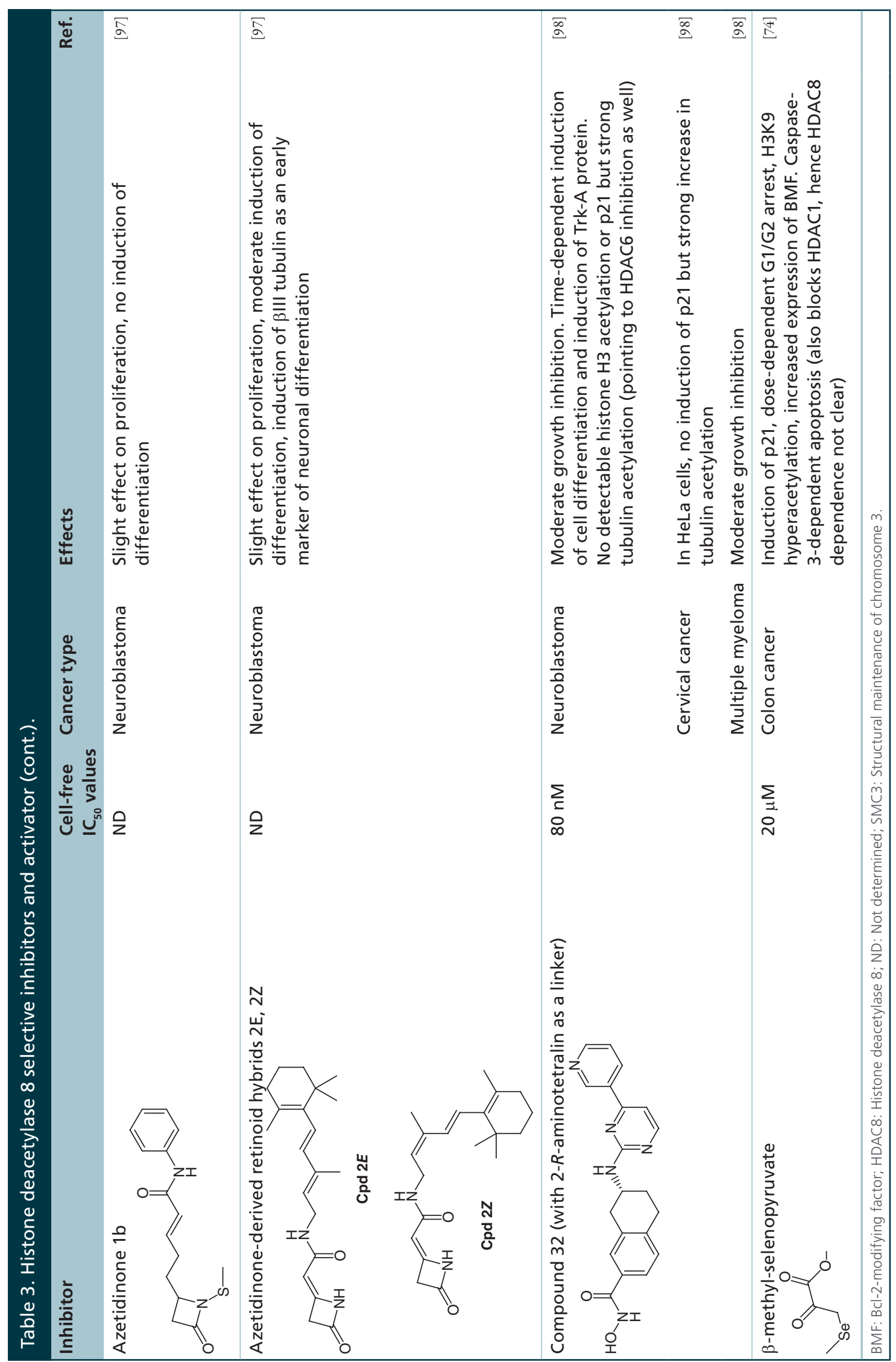




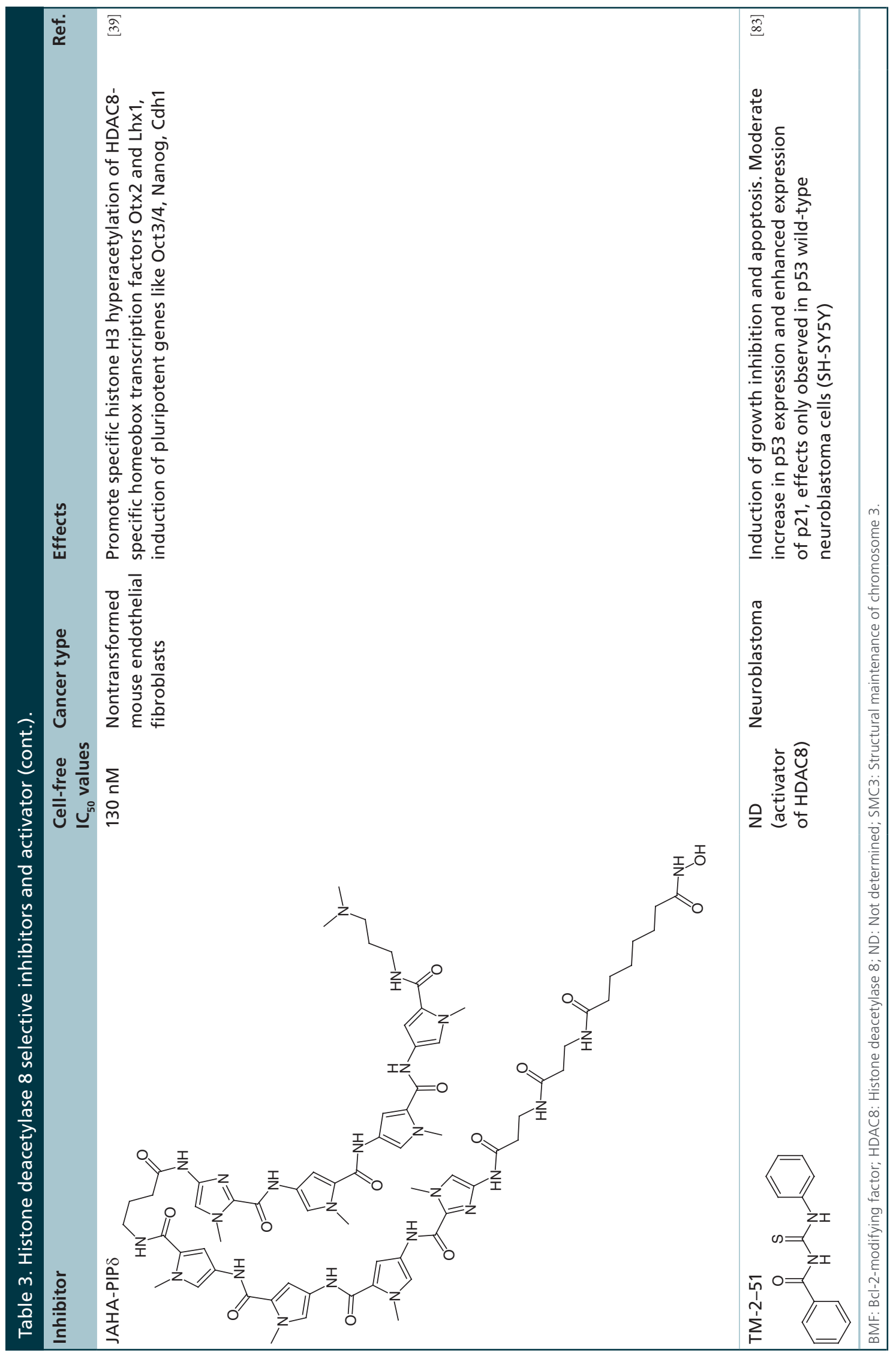


2.4-4 $\mu \mathrm{M}$ [10]. Cell toxicity was specific for T-cellderived tumors as no toxicity was observed with tumors of B-cell/monocytic/myeloid/myeloma lineage origin. Moreover, in resting or stimulated T cells PCI34051 did not cause any cell death. Interestingly, the specificity of PCI34051 toxicity to Jurkat and HuT78 cells was not accompanied by histone or tubulin acetylation [10]. AML leukemia stem cells can respond quite differently to HDAC8 inhibition. For example, PCI34051 treatment has shown moderate effects in inv(16) AML by elimination of inv(16+) AML CD34+ leukemic stem cells through reactivation of $\mathrm{p} 53$ via restoration of p53 acetylation in these cells [69]. A detailed analysis of the PCI34051-specific T-cell lymphoma effect concluded caspase-3-dependent apoptosis as a mechanism of cell death without cleavage of the proapoptotic Bid protein [10]. This relied on PLC $\gamma 1$-dependent calcium mobilization and cytochrome $\mathrm{C}$ release [10]. A quick dose-dependent increment in intracellular calcium levels was monitored at concentrations similar to those causing PLC $\gamma 1$-triggered apoptosis [10] without the requirement of T-cell receptor signaling. However, it was not clear whether PLC $\gamma 1$ was itself a direct target of HDAC8 or other adaptor proteins (of T-cell signaling pathway) that bind to activate it.

PCI34051 has lately been tested in preclinical neuroblastoma models. Treatment of neuroblastomaderived cell lines with PCI34051 or stable variants PCI48000/PCI48012 (all at $4 \mu \mathrm{M}$ ) led to significant reduction in cell numbers, increase in cell-cycle inhibitor p21WAF1/CIP1 and stimulation of cellular differentiation by upregulation of Trk-A protein followed by formation of neurofilament-positive outgrowths [77]. Application of the more stable variant PCI48012 (plasma half-life $1 \mathrm{~h}$ ) to neuroblastoma xenograft mouse models at the maximum tolerable dose of $40 \mathrm{mg} / \mathrm{kg} /$ day critically delayed neuroblastoma tumor growth and increased cellular differentiation and apoptosis as detected by caspase-3 staining of tumor samples [77]. Moreover, treatment of $\mathrm{BE}(2)-\mathrm{C}$ xenografted NMRI nude mice with PCI48012 and 13-cis retinoic acid, a clinically approved drug currently applied as part of the standard treatment combination during the maintenance phase of the treatment protocol for highrisk neuroblastoma, had a synergistic effect in retarding tumor growth and enhancing cell death [77]. The enhancement effect by PCI34051-mediated HDAC8 inhibition and retinoic acid was attributed to CREB, a protein that has been implicated in both HDAC 8 and retinoic acid signaling pathways. Of note, this compound combination was well tolerated by the animals and no hyperacetylation of tubulin or histone $\mathrm{H} 4$ in peripheral blood mononuclear cells was observed as nonspecific effects [77]. From a clinical perspective, combination of HDAC8 inhibition with retinoic acid treatment might be a promising strategy in the maintenance treatment of high-risk neuroblastoma.

PCI34051 and variants have also found application in malignant peripheral nerve sheath tumor cells [38]. Both in human (e.g., S462, STS26T, MPNST642, MPNST724) and in murine-derived cell lines (MPNST6IEPVI), PCI34051 and PCI48012 abolished cell growth and potently reduced cell viability as well as clonogenic growth capacity [38]. While an S-phase cell cycle arrest was reported in both human and murine-derived cell lines, these effects were not accompanied by tubulin nor histone $(\mathrm{H} 3 / \mathrm{H} 4)$ hyperacetylation [38]. In the MPNST6IEPVI mouse model, treatment with PCI48012 significantly diminished tumor growth and volume [38]. By using PCI34051, new target proteins in this cancer type has also been identified [38].

In addition to above-mentioned cancer types, PCI34051 has been shown to be effective in nonalcoholic fatty liver disease-associated hepatocellular carcinoma. In such cancers, HDAC8-stimulated insulin resistance and $\beta$-catenin activation [40]. Treatment of HDAC8-overexpressing cell lines BEL-7404 and PLC5 with PCI34051-induced cell death, G2-M arrest, apoptosis, $\mathrm{H} 4$ acetylation and increased expression of p53 [40]. In PLC5 cells, an additional increase in insulin sensitivity was also observed followed by a concomitant decrease in $\beta$-catenin and its downstream target CCND1 [40]. Given the application of PCI34051 and its variants in a broad spectrum of malignancies, these compounds are definitely promising leads for further preclinical evaluation.

\section{NCC149}

This inhibitor (Figure 3 \& Table 3) was identified using a rapid screen of a 151-triazole compound library generated using click chemistry [96]. The alkyne, containing a zinc-binding group, was clicked using $\mathrm{Cu}$ (I)-catalyzed azide-alkyne cycloaddition reaction to an azide forming triazoles as a linker [96]. In vitro tests revealed a low $\mathrm{IC}_{50}$ of NCC149 $(70 \mathrm{nM})$ against HDAC8 with a very high selectivity over major HDACs (nuclear extract: $\mathrm{IC}_{50} 54 \mu \mathrm{M}, \mathrm{HDACl}: \mathrm{IC}_{50}$ $38 \mu \mathrm{M}$, HDAC2 $>100 \mu \mathrm{M}$, HDAC4: $\mathrm{IC}_{50}: 44 \mu \mathrm{M}$, HDAC6: $\left.\mathrm{IC}_{50} 44 \mu \mathrm{M}\right)$. In cellulo, NCC149 induced dose-dependent acetylation of SMC3 without any significant acetylation changes in histone $\mathrm{H} 4$ indicating a selective cellular inhibition of HDAC8 [96]. This compound also exhibited cell-type specific growth inhibition with strong effects in Jurkat, HH, MT2, MT4, NB-1 and LA-N-1 but not in healthy donor peripheral blood mononuclear cells $\left(\mathrm{IC}_{50}>100 \mu \mathrm{M}\right)$. Docking of NCC149 to HDAC8 indicates that the triazole ring 
interacts with the methylene group of HDAC8 Phe152 through hydrophobic interactions. Moreover, this triazole ring seems to be pivotal for the appropriate orientation of both the hydroxamate and Phe152 binding phenylthiomethyl group contributing to potency [96]. Hence, further derivatives of NCC149 with various ring systems were designed to increase potency against HDAC8. Compound 11 (Figure 3 \& Table 3), a reversely oriented triazole, was found to be even more potent on HDAC8 with an $\mathrm{IC}_{50}$ of $53 \mathrm{nM}$ maintaining the high selectivity profile of NCC149 [67]. However, inside cells compound 11 was found to acetylate both SMC3 and $\alpha$-tubulin in a dose-dependent manner without major effects on $\mathrm{H} 3 \mathrm{~K} 9$. In contrast, a thiazole derivative exhibiting a higher $\mathrm{IC}_{50}$ value of $150 \mathrm{nM}$ (80-100-fold selectivity over other HDACs), compound 9 (Figure 3 \& Table 3), was more selective in cells in acetylating SMC3 compared with $\alpha$-tubulin or H3K9 [67]. This indicated that compound 9, though less potent on HDAC8 enzyme in vitro, could specifically inhibit HDAC8 inside cells compared with other cellular HDACs. In parity with these observations, compound 9 also showed higher growth inhibition in T-cell lymphoma cell lines (Jurkat, HH, MT4 and HuT78) compared with NCC149 [67]. This might hint to the possibility that a compact binding of HDAC8 with thiazole improves the selectivity of compound 9 over other cellular HDACs and also renders it more active inside cells.

\section{Ortho-aryl $\mathrm{N}$-hydroxycinnamides}

This series was generated using computer guided docking studies and knowledge-based design [72]. Huang et al. generated a chimeric inhibitor with the benzyl moiety of PCI34051 into a core $N$-hydroxycinnamide of different chain length derived from LBH589 [72]. An ortho-phenyl $N$-hydroxycinnamide, compound 22a (Figure 3 \& Table 3 ) with a short linker, was identified as a lead compound with similar potency as PCI34051. Further docking studies with 22a led to the insight that inclusion of additional hydrophobic groups could impart 22a better binding capacity to HDAC8. Two derivatives designed on this principle showed excellent HDAC8 inhibitory activities. Compound $22 \mathrm{~b}$ (Figure $3 \&$ Table 3 ) with a para-bromo group exhibited an $\mathrm{IC}_{50}$ of $5 \mathrm{nM}$ (tenfold more potent than PCI34051, 13-fold more potent than 22a) and 22d (Figure 3 \& Table 3) with a biphenyl warhead exhibited an $\mathrm{IC}_{50}$ of $27 \mathrm{nM}$ (twofold more potent than PCI34051, threefold more potent than 22a), both compounds being selective over other major HDACs [72]. However, only $22 \mathrm{~d}$ showed moderate growth inhibition with lung cancer cell lines. Specifically, in cell line CL1-5 with elevated HDAC8 level, 22d showed similar $\mathrm{GI}_{50}(7 \mu \mathrm{M})$ compared with SAHA $(6.2 \mu \mathrm{M})$ [72]. These effects were stronger compared with PCI34051 $\left(\mathrm{GI}_{50}>10 \mu \mathrm{M}\right) .22 \mathrm{~d}$ was nontoxic to IMR-90 fibroblasts indicating a preference toward cancer cells [72]. Moreover, this compound could also enhance chemosensitivity of resistant AML cells by specifically restoring p53 acetylation [69]. Additionally, in inv(16+) AML CD34+ leukemia stem cells, 22d-induced apoptosis [69]. In vivo, in a mouse xenograft AML model, $22 \mathrm{~d}$ significantly reduced engraftment size, AML burden and abolished leukemia inducing capacity of such leukemia stem cells [69]. Furthermore, the modulation of $22 \mathrm{~d}$ activity with a concomitant modulation of $\mathrm{p} 53$ or HDAC8 levels underlined a possibility of using this compound in combination therapy [69]. Hence, 22d is a promising lead structure to follow-up for further (pre)clinically active derivatives.

\section{Azetidinone}

An azetidinone inhibitor was designed based on the modular structure of the pan-inhibitor SAHA, belonging to the class of monocyclic- $\beta$ lactams with an azetidin-2-one ring as the zinc binding moiety [110]. Modification of the nitrogen substituents in the azetidin-2-one ring led to the identification of HDAC8selective azetidinone $1 \mathrm{~b}\left(\mathrm{IC}_{50} 4.53 \mu \mathrm{M}\right.$, other HDACs $>1000 \mu \mathrm{M})$, revealing an $N$-thiomethyl- $\beta$-lactam as a novel zinc chelating group (Figure 3) [110]. Replacement of $\mathrm{N}$-thiomethyl with $\mathrm{N}-\mathrm{H}$ or $\mathrm{N}-\mathrm{OH}$ resulted in complete loss of activity signifying the prerequisite of this group for the inhibition. Computational analysis of $1 \mathrm{~b}$ indicated that the $N$-thiomethyl-azetidin-2-one interacted with Trp141 of HDAC8 via the sulfur atom thus contributing to the specific activity [110]. This compound also showed moderate cytotoxicity $\left(\mathrm{IC}_{50}\right.$ $10-28 \mu \mathrm{M})$ in SH-SY5Y undifferentiated neuroblastoma cell line [97]. A further extension of this compound as hybrids with retinoic acid (Figure 3 \& Table 3) reduced cytotoxic potency ( $\mathrm{IC}_{50} 30-90 \mu \mathrm{M}$ range), nevertheless, imparted neural-like moderate differentiation capability by induction of $\beta$ III-tubulin [97] which again hints as using such compounds in combination therapy for neuroblastoma. However, these hybrid compounds were neither tested for HDAC8 inhibition nor for their specificity over other HDACs. Therefore, further follow-up studies are needed to define the specific mode of action.

\section{Aminotetralin-based dual inhibitors}

Phenotypic screens of acetylated tubulin and p21 induction were employed to identify the tetrahydroisoquinoline containing lead compound 12 (Figure 3 \& Table 3) as dual inhibitors of HDAC8/HDAC6 [98]. This potent inhibitor (HDAC8: $\mathrm{IC}_{50} 30 \mathrm{nM}$; HDAC6: $\mathrm{IC}_{50} 50 \mathrm{nM}$ ) exhibited high selectivity over other HDACs $(>20 \mu \mathrm{M})$ 


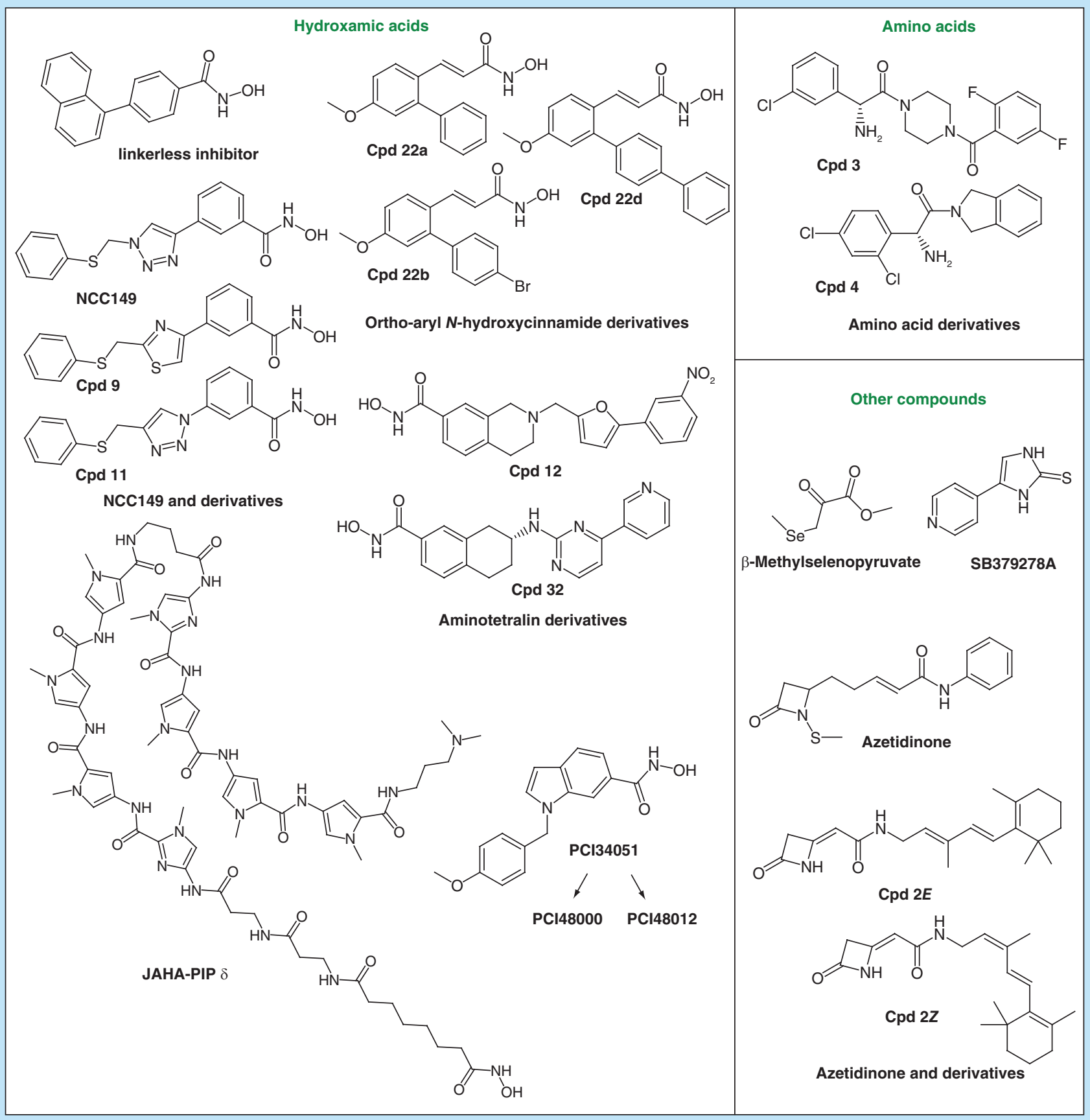

Pan inhibitors

HDAC8 activator<smiles>CC(/C=C/C(=O)NO)=C\[C@@H](C)C(=O)c1ccc(N(C)C)cc1</smiles>

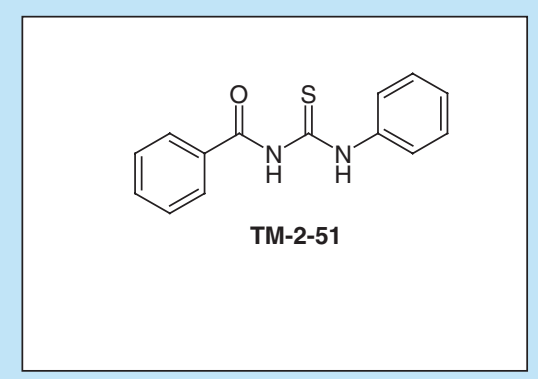


Figure 3. Inhibitors and activator of histone deacetylase 8 (see facing page). HDAC8-specific inhibitors of different zinc-binding groups (green text), pan inhibitors and a thiourea-based activator of HDA8 are shown in the figure.

Cpd: Compound; HDAC8: Histone deacetylase 8.

while inside cells, induced $70 \%$ tubulin acetylation, slight $\mathrm{p} 21$ induction and was moderately toxic $\left(\mathrm{IC}_{50}\right.$ $3.45 \mu \mathrm{M})$ [98]. However, this inhibitor had a low metabolic stability in microsomal metabolite assays due to hydrolysis of the hydroxamic acid group and $N-C$ cleavage on the tetrahydroisoquinoline moiety [98]. Moreover, the poor solubility $(>10 \mu \mathrm{g} / \mathrm{ml})$ of the inhibitor is problematic. In order to overcome these issues, new aminotetralin derivatives were designed to mimic the 2, 7-disubstituted tetrahydroisoquinoline linker. Compound 32 (Figure 3 \& Table 3) with $2-R$-aminotetralin as a linker showed the most potent inhibition with both HDAC8 $\left(\mathrm{IC}_{50} 80 \mathrm{nM}\right) / \mathrm{HDAC6}$ $\left(\mathrm{IC}_{50} 50 \mathrm{nM}\right)$ and less on other HDAC isotypes $(>30 \mu \mathrm{M})$ [98]. In cellulo, it led to hyperacetylated tubulin with an $\mathrm{EC}_{50}$ of $1.64 \mu \mathrm{M}$ but failed to induce $\mathrm{p} 21$ expression in HeLa cells [98]. In NCI-H929 (multiple myeloma) and $\mathrm{BE}(2)-\mathrm{C}$ (neuroblastoma) cells, inhibitor 32 imparted moderate toxicity with $\mathrm{GI}_{50}$ values of $7.7 \mu \mathrm{M}$ and $5.4 \mu \mathrm{M}$, respectively [98]. Nevertheless, 32 was moderately metabolically stable and soluble at $59 \mu \mathrm{g} / \mathrm{ml}$. Interestingly, 32 stimulated time-dependent differentiation such as neurite growth in $\mathrm{BE}(2)-\mathrm{C}$ cells treated at only $2 \mu \mathrm{M}$ [98]. Also, in these cells $32 \mathrm{did}$ not induce HDAC6 expression, p21 induction and $\mathrm{H} 3$ acetylation up to $10 \mu \mathrm{M}$; instead the cell differentiation marker TrkA and acetylated tubulin levels were significantly elevated [98]. With the emerging role of HDAC8 and HDAC6 in cancer such dual inhibitors can be of advantage for the treatment of cancers affected by aberrations of both enzymes. Given the fact that 32 did not induce HDAC6 expression in neuro-

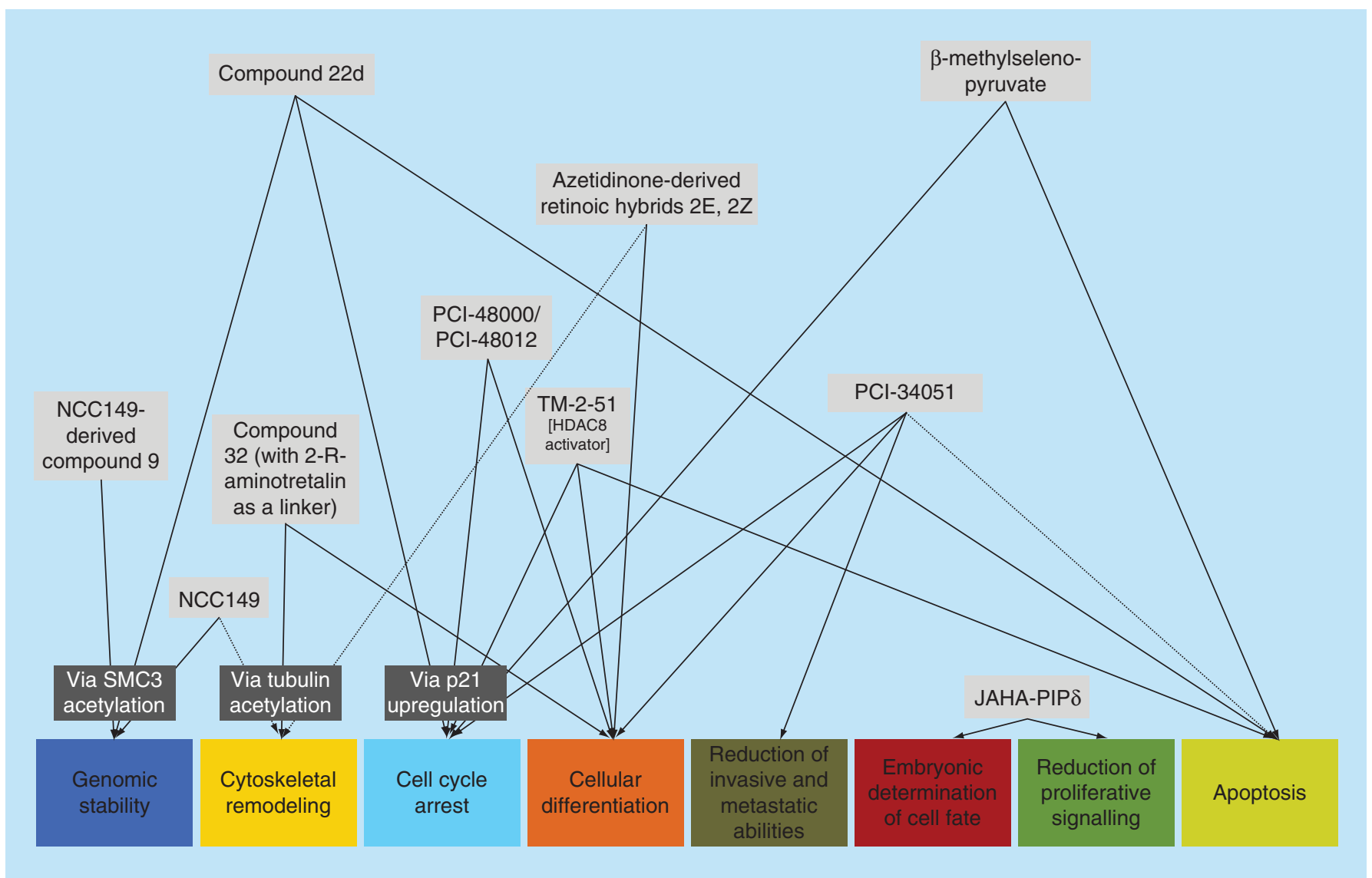

Figure 4. The hallmarks of pharmacological histone deacetylase 8 modulation. Depicted are the major cancer relevant processes targeted by selective HDAC8 modulators (inhibitors and one activator) according to studies using various cancer cell lines. Additional mechanistic information is presented for cancer hallmarks in dark gray boxes if available. Dotted arrows indicate discrepant results for different cell lines, suggesting cell line and context dependency of the respective finding. Note that the figure displays only relationships that have been experimentally addressed by the cited references. It is of course possible that additional hallmarks are influenced by a certain compound complementary to those indicated in the figure.

HDAC8: Histone deacetylase 8. 


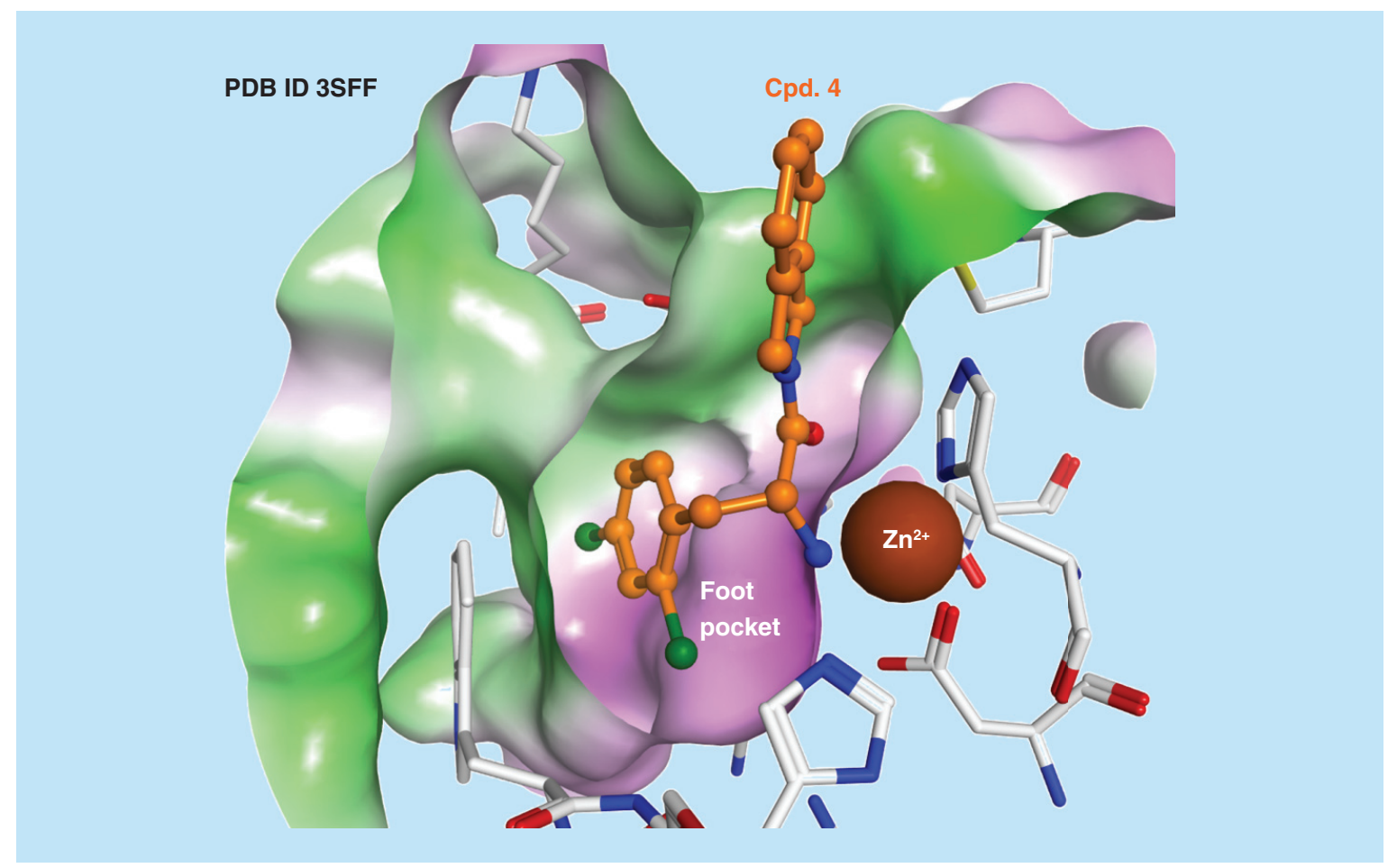

Figure 5. Crystal structure of histone deacetylase 8 in complex with an amino acid derivative (PDB ID3SFF) showing the addressing of the foot pocket.

blastoma and even stimulated differentiation, this class of compounds can be further optimized for HDAC8 specificity and would be interesting candidates to test in preclinical animal models.

\section{$\beta$-Methylselenopyruvate}

$\beta$-Methylselenopyruvate (Figure 3 \& Table 3 ) is an $\alpha$-keto-acid metabolite of natural organoselenium containing compounds like methylselenocysteine (generated by transamination), identified as a micromolar inhibitor of HDAC8 $\left(\mathrm{IC}_{50} 20 \mu \mathrm{M}\right)$ [74]. Molecular modeling suggested that this compound docked in an energetically favored orientation with the $\alpha$-carbonyl group and one of the carboxylate oxygen atoms interacting with the zinc of HDAC8 [74]. In colon cancer cells HCT116 and HT29, this inhibitor induced histone $\mathrm{H} 3$ hyperacetylation, a dose-dependent G2 $(10 \mu \mathrm{M})$ or G1 $(2 \mu \mathrm{M})$ cell cycle arrest and activation of caspases, indicative for apoptosis. An analysis of cell cycle regulator $\mathrm{p} 21$ revealed increased expression upon inhibitor treatment, hyperacetylation of histone $\mathrm{H} 3 \mathrm{~K} 9$ and $\mathrm{K} 18$ levels at P21WAF1 promoter as observed with chromatin immunoprecipitation experiments [74]. Further investigation of the apoptotic mechanism identified BMF as the key proapoptotic protein being regulated downstream by HDAC8 [73]. Additionally, this study also proposed a model stating that inhibition of HDAC8 by $\beta$-methylselenopyruvate in colon cancer cells led to dislodging of the HDAC8/STAT3 complex from the BMF promoter, recruiting $\mathrm{P} 300$ with the consequence of an elevated BMF level and activation of apoptosis [73]. Further in vivo studies will be needed to validate this theory in a physiological context. However, this inhibitor also inhibits HDAC1 and the specificity related to HDAC8-mediated downstream effects on BMF should also be proven with a specific HDAC8i as PCI34051.

\section{JAHA-PIP $\delta$}

This particular inhibitor is a hybrid of ferrocene-based SAHA analogues called Jay Amin hydroxamic acid (JAHA) with $N$-methylpyrrole (Py)- $N$-methylimidazole (Im) polyamides (PIP) [39,116]. PIPs are synthetic transcriptional modulators that mimic transcription factors by recognizing and binding to specific nucleotide sequences in the minor groove of the DNA [117]. The particular analog, JAHA-PIP $\delta$ (Figure $3 \&$ Table 3) (HDAC8: $\mathrm{IC}_{50} 130 \mathrm{nM}$ ) is a hybrid of SAHA without a capping phenyl ring with an improved PIP called PIP $\delta$ with eightfold selectivity over HDAC2 $\left(\mathrm{IC}_{50} 1.12\right.$ $\mu \mathrm{M})$ and fourfold over HDAC1 $\left(\mathrm{IC}_{50} 0.49 \mu \mathrm{M}\right)$ [39]. Notably, JAHA-PIPS enhanced endogenous acetylation of histone $\mathrm{H} 3$ in the promoter region of pluripotent genes as Oct3/4, Nanog, Cdh1 and Rex1 suggesting a role of HDAC8 in regulating pluripotency [39]. Even more strikingly, ChIP analysis revealed this analog stimulated HDAC8-specific homeobox tran- 
scription factors Otx 2 and Lhx1 promoter hyperacetylation, thus, consequently increasing the expression of these genes. HDAC8-mediated repression of Otx2 and Lhx1 is important in cell fate determination of neuronal cranial cells; while Otx2 participates in pluripotency decision, Lhx-1 determines the mesodermal differentiation [27]. This finding strongly supports the theory that HDAC8 might prefer specific gene regions in histone $\mathrm{H} 3$ for deacetylation. Also, the fact that these genes are activated in a somatic cell line like mouse embryonic fibroblasts (MEF), in which both Otx2 and Lhx-1 are not usually expressed, suggests that JAHA-PIP $\delta$ could act as modulator of cell fate. Especially, in neuroblastoma, which is linked to defects in proper sympathetic neuron maturation, the potency of this inhibitor to change cell fate might be of special interest in order to revert the neuroblastic cell type into differentiated cells. However, this assumption remains to be elucidated, since cell culture studies addressing this point with the use of JAHA-PIP $\delta$ have not been reported so far.

\section{HDAC8 activators}

\section{Cancer}

Recently, Sing et al. identified TM-2-51 (Figure 3 \& Table 3) as a selective activator of HDAC8. The basic structure is an $\mathrm{N}$-acylthiourea derivative $N$-(phenylcarbamothioyl) benzamide [118]. The pres- ence of an amide bond is essential to maintain the activating properties. Similarly, the presence of an aromatic ring connecting the other nitrogen of the thiourea also contributes to HDAC8 activation. Docking indicates clustering of this molecule at the vicinity of the active site pocket [118]. Testing TM-2-51 in p53 wild-type SH-SY5Y and p53 mutant BE(2)-C human neuroblastoma cell lines yielded surprising results with only SH-SY5Y cells showing decreased proliferation and increased apoptosis. Treatment also correlated well with the moderate increase in $\mathrm{p} 53$ level and enhanced elevation in expression of the downstream target p21 in SH-SY5Y cells [83]. Such effects were absent in BE(2)-C cell line. Although, HDAC8 regulates wild-type and mutant p53 gene expression [82] and TM-2-51 can upregulate $\mathrm{p} 53$ protein levels for both conditions, mutant p53 is more susceptible to degradation. This points to a preferential use of TM-2-51 in p53 wild-type cancers. In line, the authors propose TM-2-51 as an adjuvant to restore wild-type p53 levels in patients treated with pan HDAC inhibitors as SAHA [83].

\section{Future CdLS therapy?}

Cornelia de Lange syndrome or CdLS is a broad range of genetic disorders caused by compromised function of cohesin, hence grouped as a cohesinopathy [119]. This syndrome manifests heterogeneous phenotypes

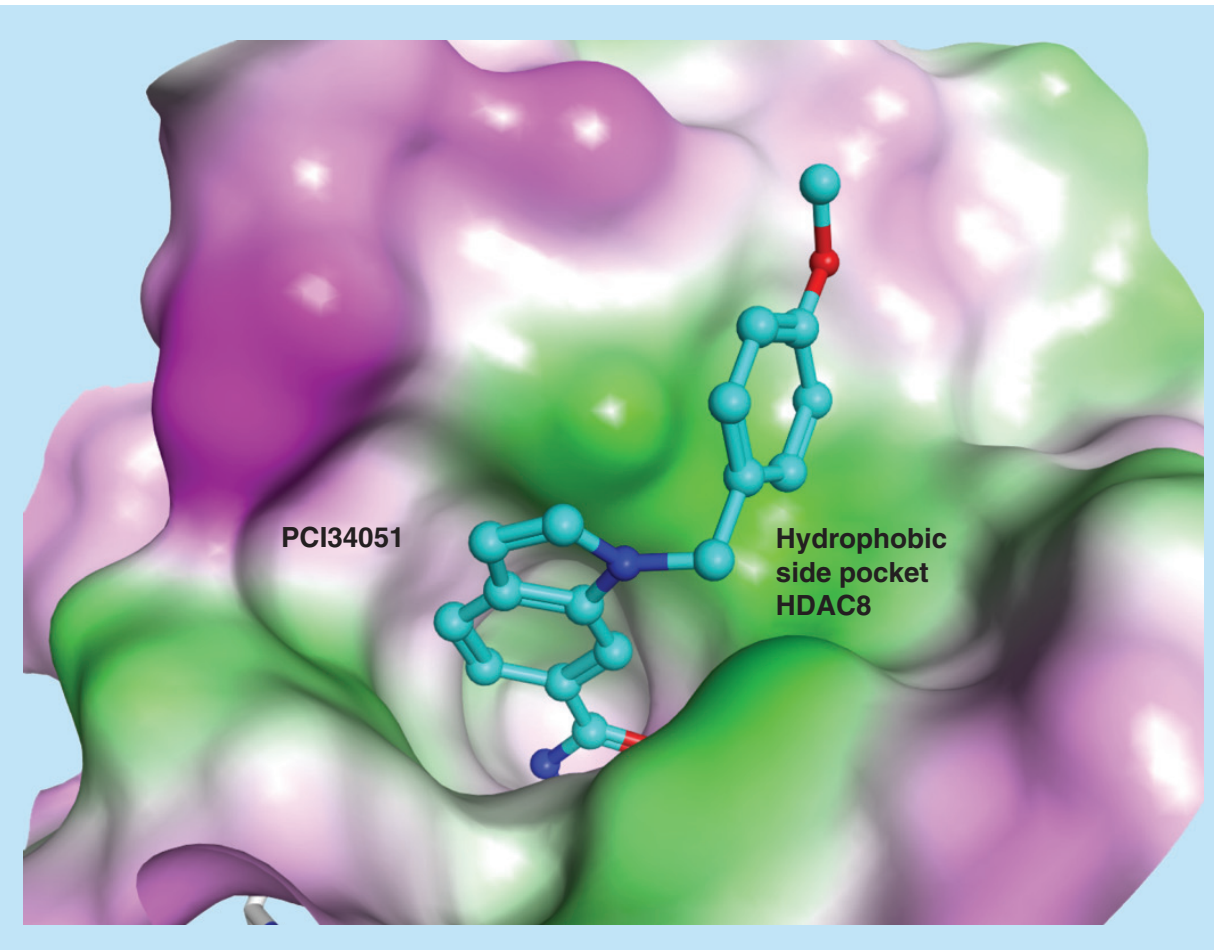

Figure 6. Docking pose of the highly selective histone deacetylase 8 inhibitor PCI34051 addressing the side pocket of histone deacetylase 8 (PDB ID 2VX5). 
affecting multiple organs with intellectual disability, distinctive facial features, growth retardation and limb malformation being the predominant symptoms [119]. The main causes of this disease are traced to genetic mutations in proteins comprising the cohesin complex, including SMC3 [120]. Along with other component proteins, SMC3 forms a clutch to hold the sister chromatids together during cell cycle progression [121]. Recently, HDAC8 has been identified as a deacetylase for SMC3 primarily deacetylating conserved lysines 105, 106 in humans [19]. This deacetylation of SMC3 is important to segregate the sister chromatids and replenish the SMC3 pool for another cell cycle event [122]. Loss of HDAC8 deacetylation activity leads to accumulation of acetylated SMC3 with decreased affinity toward chromatids ultimately effecting gene transcription [123]. Currently, several mutations in HDAC8 are linked to CdLS [19], mainly being missense and de novo aberrations [26]. Together with SMC3/SMC1A/RAD21, HDAC8 mutations account for $5 \%$ of the patients showing a heterogeneous CdLS phenotype [26].

A careful analysis of representative missense mutations revealed loss of HDAC8 activity and thermal instability in all cases $[25,124]$. Furthermore, this loss of activity was related to the occurrence of mutations in the vicinity of the active site and consequent structural changes [25]. It is also correlated to the severity of the disease or phenotype, with the most distant mutation causing less severe symptoms. Interestingly, for most mutations (that exhibited residual HDAC8 activity) enzyme activity could be rescued by treatment with TM-2-51 in a dose-dependent manner, except for the mutation located nearest to the active site $(100 \%$ inactive mutant) [25,124]. In cells expressing wild-type HDAC8 enzyme, TM-2-51 increased activity up to 12 -fold at 10 $\mu \mathrm{M}$ concentration [118]. This activator is thought to bind at two distinct sites of HDAC8 in a positive cooperative manner (as indicated from molecular docking studies), change enzyme kinetics (e.g. reduce $\mathrm{Km}$ of the substrate and markedly increase catalytic rate) in the presence of an inhibitor/substrate, and rescue catalysis [83]. Hence, the use of small-molecule activators might be used in CdLS patients with HDAC8 mutations to restore the activity of the mutant enzymes or to increase the sensitivity of HDAC8 toward the native substrates. However, the dependency on the mutation site might have a significant impact on the therapeutic outcome. Even though the present symptoms might not be completely cured, HDAC8 activators might help in slowing down the progression of the disease.

\section{Future perspective}

Until now, four HDAC inhibitors are approved by the FDA: SAHA and romidepsin for cutaneous T-cell lymphoma, belinostat for peripheral T-cell lymphoma and very recently panobinostat (LBH589) for multiple myeloma. All these drugs have a broadband inhibition profile, moderately selective for one HDAC class but not for a particular isotype. Side effects under therapy vary from nausea, fatigue, fever (pyrexia), low red blood cells (anemia), vomiting to cardiac toxicity, ventricular arrhythmia, leukopenia and thrombocytopenia. With respect to such harmful effects, isotype selective HDAC inhibitors might be advantageous to widen the therapeutic window by reducing nonspecific targeting of other HDACs and associated molecular signaling pathways. It remains to be seen, whether for example, the HDAC inhibitor chidamide, that was newly approved in China and is selective for HDAC1-3 will already provide an optimized activity/toxicity relationship [125].

To create selective HDAC8 inhibitors computational approaches have shown promising results by designing HDAC8 selective compounds, for example, based on an amino acid scaffold (Compounds 3 and 4 in Figure 3). The x-ray structures (PDB ID 3SFF and 3SFH) show that the amino and carbonyl group of the inhibitor is able to coordinate to the zinc ion whereas the terminal phenyl group is addressing the foot pocket (Figure 5). As consequence these inhibitors are highly selective for HDAC8. In addition, addressing the hydrophobic side pocket of HDAC8, for example, by inhibitor PCI34051 (Figure 3) results in a highly selective HDAC8 inhibitor. A good shape complementarity between an aromatic substituent and the side pocket is achieved (Figure 6). A similar type of interaction can be observed for example, for the linkerless HDAC8 inhibitors (Figure 3).There is strong evidence that HDAC8 is one of the causative factors for T-cell lymphoma and, hence, isotype-selective HDAC8 inhibitors present a rationale for therapies with less toxicity. Similarly, neuroblastoma-specific and selective HDAC8 inhibitors show ample promise for an improved treatment regime. As HDAC8 inhibitors can also target leukemia stem cells [69], more potent compounds can be optimized for an enhanced killing of such cells. Moreover, the possibility to combine HDAC8 inhibitors with the current treatment modalities [77] might enhance therapy in HDAC8-related cancers. HDAC8 is linked with Rad51 and a lack of radiosensitization is seen upon HDAC8 downregulation [126] which seems to point rather toward combination chemotherapeutic regimes as the route to follow. p53 modulators like leptomycin B could also offer a synergistic therapeutic effect [127]. Alternatively, small interfering RNAs or miRNAs are promising in selective HDAC8 inhibition [34]. The role of HDAC8 activators in tumors harboring wild-type p53 is also worth mentioning [83]. Due to its tissuespecific expression HDAC8-expression levels could 
be determined as a diagnostic marker for tumors as already shown for smooth muscle tumors [81]. Nevertheless, redundancy of HDACs, conserved active sites and shared protein substrates pose tremendous challenges toward isotype-specific targeting in patients. Also, in research there is a lack of preclinical animal models with a well-described dependence on HDAC8 activity. Development of new models will bring in new perspectives toward isotype selective cancer treatment. Notably, there is evidence for roles of HDAC8 in cancer progression which are not fully characterized yet and that need to be investigated further [50].

Besides cancer, HDAC8 inhibitors are attractive candidates for influenza therapy, where an explicit role of HDAC8 has been deciphered in late-penetrating viruses [31]. Moreover, HDAC8 inhibitors are currently under investigation in helminth worm infections [13,32,101]. Furthermore, new exciting results of HDAC8 activators in reversing mutant HDAC8 enzymes might open new doors for CdLS disease management. In conclusion, the field will witness a boom of research in the future years with many novel roles of HDAC8 yet to be discovered.
Financial \& competing interests disclosure

A Chakrabarti, J Melesina, W Sippl and M Jung receive funding from the European Union's Seventh Framework Programme for research, technological development and demonstration under grant agreement numbers 241865 (SEtTReND) and 602080 (AParaDDisE). M Jung (Ju295/13-1), W Sippl (Si868/13-1), O Witt (W1461/4-1) and I Oehme (Oe542/2-1) are also funded by the Deutsche Forschungsgemeinschaft (DFG). Additionally, I Oehme is supported by H.W. \& J Hector foundation \#M71. FR Kolbinger is supported by the Deutsche Krebshilfe (DKH) with a Mildred Scheel doctoral scholarship (number 112065). The authors have no other relevant affiliations or financial involvement with any organization or entity with a financial interest in or financial conflict with the subject matter or materials discussed in the manuscript apart from those disclosed.

No writing assistance was utilized in the production of this manuscript.

\section{Open access}

This work is licensed under the Attribution-NonCommercialNoDerivatives 4.0 Unported License. To view a copy of this license, visit http://creativecommons.org/licenses/by-nc$\mathrm{nd} / 4.0 /$

\section{Executive summary}

- Evolutionarily diverged from class I, histone deacetylase 8 (HDAC8) is a unique deacetylase delineating class I from class II HDACs.

Deacetylase activity \& interaction partners

- While in vitro histones and histone-derived peptides are well deacetylated, conflicting evidences from cellular studies render histones as controversial substrates.

- Though not dependent on any cofactors for activity, HDAC8 associates with many important proteins/protein complexes either to deacetylate relevant targets or acting as a scaffold for signaling events.

HDAC8 structure \& inhibitors

- Intrinsic flexible structure of HDAC8 and its similarity to other HDACs makes it a challenging target, however, the unique features of the HDAC8 binding pocket architecture allows the design of HDAC8-selective inhibitors.

Role of HDAC8 in oncogenesis

- HDAC8 is intricately involved in tumorigenesis and in addition to other tumors is a relevant target in T-cell lymphoma/leukemia and in neuroblastoma.

\section{HDAC8 inhibitors as anticancer therapeutics}

- Various isotype-selective inhibitors are identified from different methodologies and are effective in cell culture/mouse models. Dual inhibitors are also reported to be beneficial.

HDAC 8 activators

- HDAC8 activators are indicated to be used for the treatment of cancers with wild-type p53. Moreover, restoring of HDAC8 catalytic activity in cells with enzyme mutants by such activators may provide a promising strategy for disease progression management in CdLS patients.

Future perspective

- Selective targeting of HDAC8 in patients is a major challenge, however, as it offers potential for reduced side effects as compared with pan-HDAC inhibitors.

- Future perspective therapy could expand beyond cancer to viral and parasitic treatments.

\section{References}

Papers of special note have been highlighted as:

- of interest; $\bullet$ of considerable interest

1 Gerber DE. Targeted therapies: a new generation of cancer treatments. Am. Fam. Physician 77(3), 311-319 (2008).

2 Kelly WK, O'Connor OA, Krug LM et al. Phase I study of an oral histone deacetylase inhibitor, suberoylanilide hydroxamic 
acid, in patients with advanced cancer. J. Clin. Oncol. 23(17), 3923-3931 (2005)

- First FDA-approved histone deacetylase (HDAC) inhibitor.

3 Seto E, Yoshida M. Erasers of histone acetylation: the histone deacetylase enzymes. Cold Spring Harb. Perspect. Biol. 6(4), a018713 (2014).

4 You A, Tong JK, Grozinger CM, Schreiber SL. CoREST is an integral component of the CoREST- human histone deacetylase complex. Proc. Natl Acad. Sci. USA 98(4), 1454-1458 (2001).

5 Choudhary C, Kumar C, Gnad F et al. Lysine acetylation targets protein complexes and co-regulates major cellular functions. Science 325(5942), 834-840 (2009).

6 Glozak MA, Sengupta N, Zhang X, Seto E. Acetylation and deacetylation of non-histone proteins. Gene 363, 15-23 (2005).

7 Gelmetti V, Zhang J, Fanelli M, Minucci S, Pelicci PG, Lazar MA. Aberrant recruitment of the nuclear receptor corepressor-histone deacetylase complex by the acute myeloid leukemia fusion partner ETO. Mol. Cell. Biol. 18(12), 7185-7191 (1998).

8 Haberland M, Montgomery RL, Olson EN. The many roles of histone deacetylases in development and physiology: implications for disease and therapy. Nat. Rev. Genet. 10(1), 32-42 (2009).

9 Gregoretti IV, Lee YM, Goodson HV. Molecular evolution of the histone deacetylase family: functional implications of phylogenetic analysis. J. Mol. Biol. 338(1), 17-31 (2004).

10 Balasubramanian S, Ramos J, Luo W, Sirisawad M, Verner E, Buggy JJ. A novel histone deacetylase 8 (HDAC8)-specific inhibitor PCI-34051 induces apoptosis in T-cell lymphomas. Leukemia 22(5), 1026-1034 (2008).

-• Validates HDAC8 as a relevant therapeutic target in T-cell lymphomas with a low-nanomolar-specific HDAC inhibitor.

11 Oehme I, Deubzer HE, Lodrini M, Milde T, Witt O. Targeting of HDAC8 and investigational inhibitors in neuroblastoma. Expert Opin. Investig. Drugs 18(11), 1605-1617 (2009).

- Demonstration that HDAC8 expression correlates with poor prognosis in neuroblastoma.

12 Harakalova M, Van Den Boogaard MJ, Sinke R et al. $\mathrm{X}$-exome sequencing identifies a HDAC8 variant in a large pedigree with X-linked intellectual disability, truncal obesity, gynaecomastia, hypogonadism and unusual face. J. Med. Genet. 49(8), 539-543 (2012).

13 Marek M, Kannan S, Hauser AT et al. Structural basis for the inhibition of histone deacetylase 8 (HDAC8), a key epigenetic player in the blood fluke Schistosoma mansoni. PLoS Pathog. 9(9), e1003645 (2013).

14 Buggy JJ, Sideris ML, Mak P, Lorimer DD, Mcintosh B, Clark JM. Cloning and characterization of a novel human histone deacetylase, HDAC8. Biochem. J. 350 (Pt 1), 199-205 (2000).

- $\quad$ Report identification of HDAC8.

15 Van Den Wyngaert I, De Vries W, Kremer A et al. Cloning and characterization of human histone deacetylase 8. FEBS Lett. 478(1), 77-83 (2000).
- Report identification of HDAC8.

$16 \mathrm{Hu}$ E, Chen Z, Fredrickson T et al. Cloning and characterization of a novel human class I histone deacetylase that functions as a transcription repressor. J. Biol. Chem. 275(20), 15254-15264 (2000).

- $\quad$ Report identification of HDAC8.

17 Li J, Chen S, Cleary RA et al. Histone deacetylase 8 regulates cortactin deacetylation and contraction in smooth muscle tissues. Am. J. Physiol. Cell Physiol. 307(3), C288-C295 (2014).

18 Waltregny D, De Leval L, Glenisson W et al. Expression of histone deacetylase 8 , a class I histone deacetylase, is restricted to cells showing smooth muscle differentiation in normal human tissues. Am. J. Pathol. 165(2), 553-564 (2004).

19 Deardorff MA, Bando M, Nakato R et al. HDAC8 mutations in Cornelia de Lange syndrome affect the cohesin acetylation cycle. Nature 489(7415), 313-317 (2012).

-. Identification of HDAC8 as structural maintenance of chromosome 3 deacetylase.

20 Wilson BJ, Tremblay AM, Deblois G, Sylvain-Drolet G, Giguere V. An acetylation switch modulates the transcriptional activity of estrogen-related receptor alpha. Mol. Endocrinol. 24(7), 1349-1358 (2010).

21 Lee H, Sengupta N, Villagra A, Rezai-Zadeh N, Seto E. Histone deacetylase 8 safeguards the human evershorter telomeres $1 \mathrm{~B}$ (hEST1B) protein from ubiquitin mediated degradation. Mol. Cell. Biol. 26(14), 5259-5269 (2006).

22 Olson DE, Udeshi ND, Wolfson NA et al. An unbiased approach to identify endogenous substrates of "histone" deacetylase 8. ACS Chem. Biol. 9(10), 2210-2216 (2014).

-• Novel approach to identify cellular substrates of HDAC8.

23 Somoza JR, Skene RJ, Katz BA et al. Structural snapshots of human HDAC8 provide insights into the class I histone deacetylases. Structure 12(7), 1325-1334 (2004).

24 Vannini A, Volpari C, Gallinari P et al. Substrate binding to histone deacetylases as shown by the crystal structure of the HDAC8-substrate complex. EMBO Rep. 8(9), 879-884 (2007)

25 Decroos C, Bowman CM, Moser JA, Christianson KE, Deardorff MA, Christianson DW. Compromised structure and function of HDAC8 mutants identified in Cornelia de Lange Syndrome spectrum disorders. ACS Chem. Biol. 9(9), 2157-2164 (2014).

26 Kaiser FJ, Ansari M, Braunholz D et al. Loss-of-function HDAC8 mutations cause a phenotypic spectrum of Cornelia de Lange syndrome-like features, ocular hypertelorism, large fontanelle and X-linked inheritance. Hum. Mol. Genet. 23(11), 2888-2900 (2014).

27 Haberland M, Mokalled MH, Montgomery RL, Olson EN. Epigenetic control of skull morphogenesis by histone deacetylase 8. Genes Dev. 23(14), 1625-1630 (2009).

28 Fu Y, Zhang P, Ge J et al. Histone deacetylase 8 suppresses osteogenic differentiation of bone marrow stromal cells by inhibiting histone $\mathrm{H} 3 \mathrm{~K} 9$ acetylation and RUNX2 activity. Int. J. Biochem. Cell Biol. 54, 68-77 (2014). 

the innate antiviral response and beta interferon gene expression by deacetylation. Mol. Cell. Biol. 26(8), 3106-3113 (2006). mediated epigenetic reprogramming plays a key role in resistance to anthrax lethal toxin-induced pyroptosis in macrophages. J. Immunol. 193(3), 1333-1343 (2014).

31 Yamauchi Y, Boukari H, Banerjee I, Sbalzarini IF, Horvath P, Helenius A. Histone deacetylase 8 is required for centrosome cohesion and influenza A virus entry. PLoS Pathog. 7(10), e1002316 (2011).

32 Stolfa DA, Marek M, Lancelot J et al. Molecular basis for the antiparasitic activity of a mercaptoacetamide derivative that inhibits histone deacetylase 8 (HDAC8) from the human pathogen Schistosoma mansoni. J. Mol. Biol. 426(20), 3442-3453 (2014).

33 Song S, Wang Y, Xu P et al. The inhibition of histone deacetylase 8 suppresses proliferation and inhibits apoptosis in gastric adenocarcinoma. Int. J. Oncol. 47(5), 1819-1828 (2015). in human gastric adenocarcinoma and inhibits proliferation and cell cycle progression by targeting oncogene HDAC8. Target. Oncol. 11, 197-207 (2015).

35 Wolfson NA, Pitcairn CA, Fierke CA. HDAC8 substrates: histones and beyond. Biopolymers 99(2), 112-126 (2013). specificities of lysine deacetylase inhibitors in human cells. Nat. Biotechnol. 33(4), 415-423 (2015).

37 Lee H, Rezai-Zadeh N, Seto E. Negative regulation of histone deacetylase 8 activity by cyclic AMP-dependent protein kinase A. Mol. Cell. Biol. 24(2), 765-773 (2004).

38 Lopez G, Bill KL, Bid HK et al. HDAC8, a potential therapeutic target for the treatment of malignant peripheral nerve sheath tumors (MPNST). PLoS ONE 10(7), e0133302 (2015).

39 Saha A, Pandian GN, Sato S et al. Synthesis and biological evaluation of a targeted DNA-binding transcriptional activator with HDAC8 inhibitory activity. Bioorg. Med. Chem. 21(14), 4201-4209 (2013).

40 Tian Y, Wong VW, Wong GL et al. Histone deacetylase HDAC8 promotes insulin resistance and beta-catenin activation in NAFLD-associated hepatocellular carcinoma. Cancer Res. 75(22), 4803-4816 (2015).

41 Dose A, Liokatis S, Theillet FX, Selenko P, Schwarzer D. NMR profiling of histone deacetylase and acetyl-transferase activities in real time. ACS Chem. Biol. 6(5), 419-424 (2011).

42 Gurard-Levin ZA, Mrksich M. The activity of HDAC8 depends on local and distal sequences of its peptide substrates. Biochemistry 47(23), 6242-6250 (2008).

43 Wu J, Du C, Lv Z et al. The up-regulation of histone deacetylase 8 promotes proliferation and inhibits apoptosis in hepatocellular carcinoma. Dig. Dis. Sci. 58(12), 3545-3553 (2013).

44 Alam N, Zimmerman L, Wolfson NA, Joseph CG, Fierke CA, Schueler-Furman O. Structure-based identification of HDAC8 non-histone substrates. Structure 24(3), 458-468 (2016).
45 Durst KL, Lutterbach B, Kummalue T, Friedman AD, Hiebert SW. The inv(16) fusion protein associates with corepressors via a smooth muscle myosin heavychain domain. Mol. Cell. Biol. 23(2), 607-619 (2003).

46 Gao J, Siddoway B, Huang Q, Xia H. Inactivation of CREB mediated gene transcription by HDAC 8 bound protein phosphatase. Biochem. Biophys. Res. Commun. 379(1), 1-5 (2009).

47 Qian Y, Zhang J, Jung YS, Chen X. DEC1 coordinates with HDAC8 to differentially regulate TAp73 and DeltaNp73 expression. PLoS ONE 9(1), e84015 (2014).

48 Karolczak-Bayatti M, Sweeney M, Cheng J et al. Acetylation of heat shock protein 20 (Hsp20) regulates human myometrial activity. J. Biol. Chem. 286(39), 34346-34355 (2011).

49 Waltregny D, Glenisson W, Tran SL et al. Histone deacetylase HDAC8 associates with smooth muscle alphaactin and is essential for smooth muscle cell contractility. FASEB J. 19(8), 966-968 (2005).

50 Chao MW, Chu PC, Chuang HC et al. Non-epigenetic function of HDAC8 in regulating breast cancer stem cells by maintaining Notch1 protein stability. Oncotarget 7(2), 1796-1807 (2015).

51 Chakrabarti A, Oehme I, Witt O et al. HDAC8: a multifaceted target for therapeutic interventions. Trends Pharmacol. Sci. 36(7), 481-492 (2015).

-. Overview of HDAC8 as a therapeutic target.

52 Schapira M. Structural biology of human metal-dependent histone deacetylases; histone deacetylases: the biology and clinical implication. In: Handbook of Experimental Pharmacology. Yao T, Seto E (Eds). Springer, Berlin, Heidelberg, Germany 225-240 (2011).

53 Melesina J, Robaa D, Pierce RJ, Romier C, Sippl W. Homology modeling of parasite histone deacetylases to guide the structure-based design of selective inhibitors. J. Mol. Graph. Model. 62, 342-361 (2015).

54 Micelli C, Rastelli G. Histone deacetylases: structural determinants of inhibitor selectivity. Drug Discov. Today 20(6), 718-735 (2015).

55 Krennhrubec K, Marshall BL, Hedglin M, Verdin E, Ulrich SM. Design and evaluation of 'linkerless' hydroxamic acids as selective HDAC8 inhibitors. Bioorg. Med. Chem. Lett. 17(10), 2874-2878 (2007).

56 Olson DE, Wagner FF, Kaya T et al. Discovery of the first histone deacetylase $6 / 8$ dual inhibitors. J. Med. Chem. 56(11), 4816-4820 (2013).

57 Nakagawa M, Oda Y, Eguchi T et al. Expression profile of class I histone deacetylases in human cancer tissues. Oncol. Rep. 18(4), 769-774 (2007).

58 Oehme I, Deubzer HE, Wegener D et al. Histone deacetylase 8 in neuroblastoma tumorigenesis. Clin. Cancer Res. 15(1), 91-99 (2009)

- Demonstration that HDAC8 expression correlates with poor prognosis in neuroblastoma.

59 Wilmott JS, Colebatch AJ, Kakavand $\mathrm{H}$ et al. Expression of the class 1 histone deacetylases HDAC 8 and 3 are associated with improved survival of patients with metastatic melanoma. Mod. Pathol. 28(7), 884-894 (2015). 
60 Park SY, Jun JA, Jeong KJ et al. Histone deacetylases 1, 6 and 8 are critical for invasion in breast cancer. Oncol. Rep. 25(6), 1677-1681 (2011).

61 Vannini A, Volpari C, Filocamo G et al. Crystal structure of a eukaryotic zinc-dependent histone deacetylase, human HDAC8, complexed with a hydroxamic acid inhibitor. Proc. Natl Acad. Sci. USA 101(42), 15064-15069 (2004).

- Structural analysis of mammalian HDAC8 in complex with HDAC inhibitors.

62 Higuchi T, Nakayama T, Arao T, Nishio K, Yoshie O. SOX4 is a direct target gene of FRA-2 and induces expression of HDAC8 in adult T-cell leukemia/lymphoma. Blood 121(18), 3640-3649 (2013).

63 Schilham MW, Oosterwegel MA, Moerer P et al. Defects in cardiac outflow tract formation and pro-B-lymphocyte expansion in mice lacking Sox-4. Nature 380(6576), 711-714 (1996).

64 Potzner MR, Tsarovina K, Binder E et al. Sequential requirement of Sox 4 and Sox11 during development of the sympathetic nervous system. Development 137(5), 775-784 (2010).

65 Bergsland M, Werme M, Malewicz M, Perlmann T, Muhr J. The establishment of neuronal properties is controlled by Sox 4 and Sox11. Genes Dev. 20(24), 3475-3486 (2006).

66 R2: Genomics Analysis and Visualization Platform. http://r2.amc.nl

67 Suzuki T, Muto N, Bando M et al. Design, synthesis, and biological activity of NCC149 derivatives as histone deacetylase 8-selective inhibitors. ChemMedChem 9(3), 657-664 (2014).

68 Bradbury C, Khanim F, Hayden R et al. Histone deacetylases in acute myeloid leukaemia show a distinctive pattern of expression that changes selectively in response to deacetylase inhibitors. Leukemia 19(10), 1751-1759 (2005).

69 Qi J, Singh S, Hua WK et al. HDAC8 inhibition specifically targets $\operatorname{Inv}(16)$ acute myeloid leukemic stem cells by restoring p53 acetylation. Cell Stem Cell 17(5), 597-610 (2015).

- Demonstrates that specific inhibition of HDAC8 abrogates leukemic stem cells and delays acute myeloid leukemia.

70 Gao SM, Chen CQ, Wang LY et al. Histone deacetylases inhibitor sodium butyrate inhibits JAK2/STAT signaling through upregulation of SOCS1 and SOCS3 mediated by HDAC8 inhibition in myeloproliferative neoplasms. Exp. Hematol. 41(3), 261-270 (2013).

71 Osada H, Tatematsu Y, Saito H, Yatabe Y, Mitsudomi T, Takahashi T. Reduced expression of class II histone deacetylase genes is associated with poor prognosis in lung cancer patients. Int. J. Cancer 112(1), 26-32 (2004).

72 Huang WJ, Wang YC, Chao SW et al. Synthesis and biological evaluation of ortho-aryl $\mathrm{N}$-hydroxycinnamides as potent histone deacetylase (HDAC) 8 isoformselective inhibitors. ChemMedChem 7(10), 1815-1824 (2012).

73 Kang Y, Nian H, Rajendran P et al. HDAC8 and STAT3 repress BMF gene activity in colon cancer cells. Cell Death Dis. 5, e1476 (2014).

74 Nian H, Bisson WH, Dashwood WM, Pinto JT, Dashwood RH. Alpha-keto acid metabolites of organoselenium compounds inhibit histone deacetylase activity in human colon cancer cells. Carcinogenesis 30 (8), 1416-1423 (2009).

75 Lucio-Eterovic AK, Cortez MA, Valera ET et al. Differential expression of 12 histone deacetylase (HDAC) genes in astrocytomas and normal brain tissue: class II and IV are hypoexpressed in glioblastomas. BMC Cancer 8, 243 (2008).

76 Hsieh CL, Ma HP, Su CM et al. Alterations in histone deacetylase 8 lead to cell migration and poor prognosis in breast cancer. Life Sci. 151, 7-14 (2016).

77 Rettig I, Koeneke E, Trippel F et al. Selective inhibition of HDAC8 decreases neuroblastoma growth in vitro and in vivo and enhances retinoic acid-mediated differentiation. Cell Death Dis. 6, e1657 (2015).

- Demonstration of HDAC8 inhibition in mouse preclinical neuroblastoma models and possibility of combination therapy with retinoic acids.

78 Milde T, Oehme I, Korshunov A et al. HDAC5 and HDAC9 in medulloblastoma: novel markers for risk stratification and role in tumor cell growth. Clin. Cancer Res. 16(12), 3240-3252 (2010).

79 Waltregny D, North B, Van Mellaert F, De Leval J, Verdin E, Castronovo V. Screening of histone deacetylases (HDAC) expression in human prostate cancer reveals distinct class I HDAC profiles between epithelial and stromal cells. Eur. J. Histochem. 48(3), 273-290 (2004).

80 Lehmann M, Hoffmann MJ, Koch A, Ulrich SM, Schulz WA, Niegisch G. Histone deacetylase 8 is deregulated in urothelial cancer but not a target for efficient treatment. J. Exp. Clin. Cancer Res. 33(1), 59 (2014).

81 De Leval L, Waltregny D, Boniver J, Young RH, Castronovo $\mathrm{V}$, Oliva E. Use of histone deacetylase 8 (HDAC8), a new marker of smooth muscle differentiation, in the classification of mesenchymal tumors of the uterus. Am. J. Surg. Pathol. 30(3), 319-327 (2006).

82 Yan W, Liu S, Xu E, Zhang J, Zhang Y, Chen X. Histone deacetylase inhibitors suppress mutant $\mathrm{p} 53$ transcription via histone deacetylase 8. Oncogene 32 (5), 599-609 (2013).

83 Singh RK, Cho K, Padi SK et al. Mechanism of $\mathrm{N}$-Acylthiourea-mediated activation of human histone deacetylase 8 (HDAC8) at molecular and cellular levels. J. Biol. Chem. 290 (10), 6607-6619 (2015).

84 Killick R, Niklison-Chirou M, Tomasini R et al. p73: a multifunctional protein in neurobiology. Mol. Neurobiol. 43(2), 139-146 (2011).

85 Rufini A, Agostini M, Grespi F et al. p73 in cancer. Genes Cancer 2(4), 491-502 (2011).

86 Qian Y, Zhang J, Yan B, Chen X. DEC1, a basic helixloop-helix transcription factor and a novel target gene of the p 53 family, mediates $\mathrm{p} 53$-dependent premature senescence. J. Biol. Chem. 283(5), 2896-2905 (2008).

87 He J, Liu H, Chen Y. Effects of trichostatin A on HDAC8 expression, proliferation and cell cycle of Molt- 4 cells. J. Huazhong Univ. Sci. Technolog. Med. Sci. 26(5), 531-533 (2006).

88 Wu S, Luo Z, Yu PJ, Xie H, He YW. Suberoylanilide hydroxamic acid (SAHA) promotes the epithelial mesenchymal transition of triple negative breast cancer 
cells via HDAC8/FOXA1 signals. Biol. Chem. 397(1), 75-83 (2015).

89 Lodrini M, Oehme I, Schroeder C et al. MYCN and HDAC2 cooperate to repress miR-183 signaling in neuroblastoma. Nucleic Acids Res. 41(12), 6018-6033 (2013).

90 Oehme I, Linke JP, Bock BC et al. Histone deacetylase 10 promotes autophagy-mediated cell survival. Proc. Natl Acad. Sci. USA 110(28), E2592-E2601 (2013).

91 Koeneke E, Witt O, Oehme I. HDAC family members intertwined in the regulation of autophagy: a druggable vulnerability in aggressive tumor entities. Cells 4(2), 135-168 (2015).

92 Guida N, Laudati G, Galgani M et al. Histone deacetylase 4 promotes ubiquitin-dependent proteasomal degradation of Sp3 in SH-SY5Y cells treated with di(2-ethylhexyl) phthalate (DEHP), determining neuronal death. Toxicol. Appl. Pharmacol. 280 (1), 190-198 (2014).

93 Witt O, Deubzer HE, Lodrini M, Milde T, Oehme I. Targeting histone deacetylases in neuroblastoma. Curr. Pharm. Des. 15(4), 436-447 (2009).

94 Witt O, Deubzer HE, Milde T, Oehme I. HDAC family: what are the cancer relevant targets? Cancer Lett. 277(1), 8-21 (2009).

95 Hu E, Dul E, Sung CM et al. Identification of novel isoformselective inhibitors within class I histone deacetylases. J. Pharmacol. Exp. Ther. 307(2), 720-728 (2003).

96 Suzuki T, Ota Y, Ri M et al. Rapid discovery of highly potent and selective inhibitors of histone deacetylase 8 using click chemistry to generate candidate libraries. J. Med. Chem. 55(22), 9562-9575 (2012).

97 Pori M, Galletti P, Soldati R, Calza L, Mangano C, Giacomini D. Azetidinone-retinoid hybrids: synthesis and differentiative effects. Eur. J. Med. Chem. 70, 857-863 (2013).

98 Tang G, Wong JC, Zhang W et al. Identification of a novel aminotetralin class of HDAC6 and HDAC 8 selective inhibitors. J. Med. Chem. 57(19), 8026-8034 (2014).

Whitehead L, Dobler MR, Radetich B et al. Human HDAC isoform selectivity achieved via exploitation of the acetate release channel with structurally unique small molecule inhibitors. Bioorg. Med. Chem. 19(15), 4626-4634 (2011).

100 Cao GP, Arooj M, Thangapandian S et al. A lazy learningbased QSAR classification study for screening potential histone deacetylase 8 (HDAC8) inhibitors. SAR QSAR Environ. Res. 26(5), 397-420 (2015).

101 Kannan S, Melesina J, Hauser A et al. Discovery of inhibitors of schistosoma mansoni HDAC8 by combining homology modeling, virtual screening and in vitro validation. J. Chem. Inf. Model. 54(10), 3005-3019 (2014).

102 Wu R, Lu Z, Cao Z, Zhang Y. Zinc chelation with hydroxamate in histone deacetylases modulated by water access to the linker binding channel. J. Am. Chem. Soc. 133(16), 6110-6113 (2011).

103 Wu R, Wang S, Zhou N, Cao Z, Zhang Y. A protonshuttle reaction mechanism for histone deacetylase 8 and the catalytic role of metal ions. J. Am. Chem. Soc. 132(27), 9471-9479 (2010).

104 Huang D, Li X, Xiu Z. Molecular modeling of the interactions between histone deacetylase 8 and inhibitors. J. Theor. Comput. Chem. 11(4), 907-924 (2012).

105 Kunze MB, Wright DW, Werbeck ND, Kirkpatrick J, Coveney PV, Hansen DF. Loop interactions and dynamics tune the enzymatic activity of the human histone deacetylase 8. J. Am. Chem. Soc. 135(47), 17862-17868 (2013).

106 Tang W, Luo T, Greenberg EF, Bradner JE, Schreiber SL. Discovery of histone deacetylase 8 selective inhibitors. Bioorg. Med. Chem. Lett. 21(9), 2601-2605 (2011).

107 Giannini G, Marzi M, Pezzi R et al. N-hydroxy-(4-oxime)cinnamide: a versatile scaffold for the synthesis of novel histone deacetylase [correction of deacetilase] (HDAC) inhibitors. Bioorg. Med. Chem. Lett. 19(8), 2346-2349 (2009).

108 Lobera M, Madauss KP, Pohlhaus DT et al. Selective class IIa histone deacetylase inhibition via a nonchelating zincbinding group. Nat. Chem. Biol. 9(5), 319-325 (2013).

109 Heimburg T, Chakrabarti A, Lancelot J et al. Structurebased design and synthesis of novel inhibitors targeting HDAC8 from schistosoma mansoni for the treatment of schistosomiasis. J. Med. Chem. 59(6), 2423-2435 (2016).

110 Galletti P, Quintavalla A, Ventrici C et al. Azetidinones as zinc-binding groups to design selective HDAC8 inhibitors. ChemMedChem 4(12), 1991-2001 (2009).

111 Gupta SP. QSAR studies on hydroxamic acids: a fascinating family of chemicals with a wide spectrum of activities. Chem. Rev. 115(13), 6427-6490 (2015).

112 Day JA, Cohen SM. Investigating the selectivity of metalloenzyme inhibitors. J. Med. Chem. 56(20), 7997-8007 (2013).

113 Wagner FF, Olson DE, Gale JP et al. Potent and selective inhibition of histone deacetylase 6 (HDAC6) does not require a surface-binding motif. J. Med. Chem. 56(4), 1772-1776 (2013).

114 De Vreese R, Van Steen N, Verhaeghe T et al. Synthesis of benzothiophene-based hydroxamic acids as potent and selective HDAC6 inhibitors. Chem. Commun. 51(48), 9868-9871 (2015).

115 Atadja P. Development of the pan-DAC inhibitor panobinostat (LBH589): successes and challenges. Cancer Lett. 280 (2), 233-241 (2009).

116 Spencer J, Amin J, Wang M et al. Synthesis and biological evaluation of JAHAs: ferrocene-based histone deacetylase inhibitors. ACS Med. Chem. Lett. 2(5), 358-362 (2011).

117 Buchmueller KL, Staples AM, Howard CM et al. Extending the language of DNA molecular recognition by polyamides: unexpected influence of imidazole and pyrrole arrangement on binding affinity and specificity. J. Am. Chem. Soc. 127(2), 742-750 (2005).

118 Singh RK, Mandal T, Balsubramanian N et al. Histone deacetylase activators: $\mathrm{N}$-acetylthioureas serve as highly potent and isozyme selective activators for human histone deacetylase- 8 on a fluorescent substrate. Bioorg. Med. Chem. Lett. 21(19), 5920-5923 (2011). 
119 Boyle MI, Jespersgaard C, Brondum-Nielsen K, Bisgaard AM, Tumer Z. Cornelia de Lange syndrome. Clin. Genet. 88(1), 1-12 (2015).

120 Deardorff MA, Kaur M, Yaeger D et al. Mutations in cohesin complex members SMC3 and SMC1A cause a mild variant of Cornelia de Lange syndrome with predominant mental retardation. Am. J. Hum. Genet. 80 (3), 485-494 (2007).

121 Gligoris TG, Scheinost JC, Burmann F et al. Closing the cohesin ring: structure and function of its Smc3-kleisin interface. Science 346(6212), 963-967 (2014).

122 Borges V, Lehane C, Lopez-Serra L et al. Hos1 deacetylates Smc3 to close the cohesin acetylation cycle. Mol. Cell 39(5), 677-688 (2010).

123 Mannini L, Fabien CL, Cucco F et al. Mutant cohesin affects RNA polymerase II regulation in Cornelia de Lange syndrome. Sci. Rep. 5, 16803 (2015).
124 Decroos C, Christianson NH, Gullett LE et al. Biochemical and structural characterization of HDAC8 mutants associated with Cornelia de Lange syndrome spectrum disorders. Biochemistry 54(42), 6501-6513 (2015).

125 Shi Y, Dong M, Hong X et al. Results from a multicenter, open-label, pivotal Phase II study of chidamide in relapsed or refractory peripheral T-cell lymphoma. Ann. Oncol. 26(8), 1766-1771 (2015)

$126 \mathrm{Kim} \mathrm{JH}$, Moon SH, No M et al. Isotype-specific inhibition of histone deacetylases: identification of optimal targets for radiosensitization. Cancer Res. Treat. 48(3), 1130-1140 (2016).

127 Kim IA, Shin JH, Kim IH et al. Histone deacetylase inhibitor-mediated radiosensitization of human cancer cells: class differences and the potential influence of p53. Clin. Cancer Res. 12 (3 Pt 1), 940-949 (2006). 